Mon. Not. R. Astron. Soc. 000, 1-20 (2016) Printed 25 September $2018 \quad$ (MN LATEX style file v2.2)

\title{
The Pulse Luminosity Function of Swift Gamma-ray Bursts
}

\author{
A. Amaral-Rogers ${ }^{1 \star}$, R. Willingale ${ }^{1}$ and P.T. O'Brien ${ }^{1}$ \\ ${ }^{1}$ Department of Physics and Astronomy, University of Leicester, LE1 7RH, UK
}

Accepted 2016 September 19. Received 2016 August 28; in original form 2016 March 7

\begin{abstract}
The complete Swift BAT and XRT light curves of 118 GRBs with known redshifts were fitted using the physical model of GRB pulses by Willingale et al. (2010) to produce a total of 607 pulses. We compute the pulse luminosity function utilising three GRB formation rate models: a progenitor that traces the cosmic star formation rate density (CSFRD) with either a single population of GRBs, coupled to various evolutionary parameters, or a bimodal population of high and low luminosity GRBs; and a direct fit to the GRB formation rate excluding any a-priori assumptions.

We find that a single population of GRB pulses with an evolving luminosity function is preferred over all other univariate evolving GRB models, or bimodal luminosity functions in reproducing the observed GRB pulse L-z distribution and that the magnitude of the evolution in brightness is consistent with studies that utilise only the brightest GRB pulses. We determine that the appearance of a GRB formation rate density evolution component is an artifact of poor parameterisation of the CSFRD at high redshifts rather than indicating evolution in the formation rate of early epoch GRBs. We conclude that the single brightest region of a GRB lightcurve holds no special property; by incorporating pulse data from the totality of GRB emission we boost the GRB population statistics by a factor of 5 , rule out some models utilised to explain deficiencies in GRB formation rate modelling, and constrain more tightly some of the observed parameters of GRB behaviour.
\end{abstract}

\section{INTRODUCTION}

The luminosity function (LF) is a powerful tool for population analysis and, when applied to Gamma-ray bursts (GRBs), is used to verify theoretical models of the physical processes that go into forming GRBs; and as a benchmark for observation rates of future GRB missions, and gravitational wave detection likelihoods. The luminosity function does however require a precise measure of the distance to the GRB in order to convert from the observed flux to the restframe luminosity. In the era of Swift observations, over one thousand GRBs have been observed with approximately $1 / 3$ having an associated redshift, meaning that GRB luminosity functions are currently built upon relatively small sample sizes compared to other luminosity functions found in astrophysics. The standard technique for generating a luminosity function for GRBs is to utilise either a time-averaged luminosity, or the brightest part of a burst, as the characteristic luminosity of the GRB; and in such cases where there is little, or no, variation in the lightcurve such an approach is acceptable. The majority of Swift GRBs, however, show significant variation in their lightcurves with multiple peaks in the early prompt and late-time emission that, in some cases, are of comparable brightness to the most luminous part of the GRB lightcurve. In this paper we follow a different approach. Using a physically motivated model for the prompt and high-latitude emission from GRBs (Genet \& Granot 2009; Willingale et al. 2010) we fit the lightcurves of 118 long
Gamma-ray bursts (LGRBs) to produce a total of 607 individual Gamma-ray pulses and X-ray flares. The wealth of information stored within these other, less-luminous, pulses are utilised to produce a GRB pulse luminosity function; of which the more conventional LGRB LF can be considered as a high-luminosity subset. As with papers investigating the GRB luminosity function, we find discrepencies between observed, and theoretical LF models that require additional evolutionary effects to correct for. In this paper we evaluate extensive solutions to these discrepencies: additional luminosity, rate, and metallicity density evolution; bimodal low/high luminosity functions; and direct fitting of GRB formation rates. We find that in all cases a model that incorporates evolution in the break luminosity, such that higher redshift GRBs are more luminous, is preferred over one that does not. We also do not see strong evidence of a divergence between the cosmic star formation rate density and the GRB formation rate, nor any compelling evidence of a separate population of high and low luminosity GRBs. Our findings are broadly consistent with GRB LF studies whilst producing better defined evolutionary parameters, suggesting that there is nothing special about the single brightest pulse and that studies into GRB population behaviour should include all the pulse information available.

Prior to the launch of Swift (Gehrels et al. 2004), the number of LGRBs localised to a suitably fine error circle on the sky such that follow-up observation could find an asso- 
ciated host galaxy or afterglow was small; out of some 2704 GRBs detected by BATSE (Goldstein et al. 2013) only a handful had a measured redshift, made possible only due to simultaneous detection of the burst by other Gammaray missions with greater localisation abilities. Given the lack of real redshifts, many authors instead sought to derive pseudo-redshifts using properties of the LGRB lightcurves in order to derive a LGRB luminosity function; the most popular of which included the lag-luminosity relationship (Norris, Marani \& Bonnell 2000; Kocevski \& Liang 2006), the variability-luminosity relationship (Fenimore \& RamirezRuiz 2000; Reichart et al. 2001; Lloyd-Ronning, Fryer \& Ramirez-Ruiz 2002; Wei \& Gao 2003); and the Amati relationship (Amati et al. 2002; Atteia 2003; Firmani et al. 2004; Yonetoku et al. 2004; Salvaterra \& Chincarini 2007; Salvaterra et al. 2009). The large intrinsic scatter within these relationships produces however a redshift distribution that, whilst arguably it represents that of the true LGRB redshift distribution, also shows significant uncertainty in the fitted parameters. With the launch of the Swift mission, with its fast slew rate and accurate on-sky localisation, suddenly a large proportion of GRBs being detected had associated photometric and/or spectroscopic redshifts of either the host galaxy or the GRB's X-ray afterglow within a day or two of initial observation. Early Swift GRB LF papers continued to develop the LGRB luminosity function by utilising either small numbers of LGRBs with measured redshifts (Li 2008; Kistler et al. 2008), with poor constraints on fitted parameters; or by artificially boosting the LGRB redshift sample by combining real and pseudo redshift data from Swift and BATSE (Butler, Bloom \& Poznanski 2010; Salvaterra et al. 2012). Over time, the sample size of GRBs with observed redshifts has increased ${ }^{1}$ and contemporary GRB LF studies utilise larger datasets with entirely observed redshifts (Wanderman \& Piran 2010; Cao et al. 2011; Robertson \& Ellis 2012; Howell \& Coward 2013; G. Dainotti et al. 2014; Petrosian, Kitanidis \& Kocevski 2015; Pescalli et al. 2015; Yu et al. 2015; Deng et al. 2016).

Throughout these earlier studies the emphasis has been on trying to extract information about the average behaviour of the LGRB as a whole; in general, characterising the luminosity of a GRB using the flux of the single brightest peak in the lightcurve binned in 1 second bins (see for example Yonetoku et al. (2004)). In reality the lightcurve of a GRB is complex and the scale of variability between lightcurves is bewildering. The luminosity and total energy output of GRBs spans many orders of magnitude. Whilst some bursts consist of a single Fast Rise Exponential Decay (FRED) profile, others have multiple peaks; some are very spikey with rapid variations while others have a much smoother profile. Many lightcurves display astonishing chaotic time-variability, continually varying between bright, short peaks and low troughs where in some cases the flux drops below the detection threshold for a while before flaring up again. The current paradigm is of the totality of the prompt emission constructed of simple pulses (Norris et al. 2005; Willingale et al. 2010). Lasting from fractions of a second, to minutes in duration, these pulses are indepen-

1 As of January 2016 over 1000 GRBs have been observed by Swift with 295 GRBs having associated redshifts. dent of each other but with many overlapping to some degree to produce the incredible lightcurve variation observed. Late-time X-ray flares (Chincarini et al. 2007; Falcone et al. 2007) seen above the afterglow emission hundreds, and in some cases thousands of seconds, after the initial trigger appear to follow the same mechanism as the prompt emission pulses and can be considered as the lower energy tail of a unimodal pulse energy distribution ${ }^{2}$. Given the wealth of information contained within GRB lightcurves, the approach taken by the authors is to fit each individual pulse using a physical model characterised by a few simple parameters: the peak flux, a characteristic time scale, a rise time as a fraction of the characteristic time scale, and a spectral shape; the model furthermore incorporates spectral evolution such that the rise and decay to and from the peak of each pulse depend on the changing X-ray spectrum. Instead of the single data point extracted by more conventional GRB luminosity function studies, we fit, on average, 5 pulses per GRB, with the more variable lightcurves containing pulses numbering in the tens, significantly increasing our sample size over single pulse studies. Using the measured redshift, the peak flux, and spectrum we derive the rest-frame bolometric luminosity for each pulse, and use the totality of our data to construct and evaluate various GRB pulse luminosity functions.

The structure of this paper is therefore as follows: we discuss the selection criteria for our GRB sample; the physical model for pulses, and flares; and the fitting technique in Section 2. We outline the various methods for constructing a luminosity function in Section 3; and in Section 4 we discuss the Markov Chain Monte Carlo (MCMC) routine utilised to fit our luminosity function model parameters. Sections 5, 6, and 7 are discussions on the results from models convolved to the cosmic star formation rate density with either: a single population of GRB progenitors (Type I models), or two separate populations of high, and low luminosity GRBs (Type II models); and GRB formation rate models that rely on no prior assumptions on progenitor mechanisms (type III models). Section 8 compares the LGRB formation rates with the observed cosmic star formation rate density and we finally conclude our findings in Section 9.

\section{LIGHTCURVE FITTING}

We analysed the lightcurves of LGRBs with associated redshifts observed by both the Swift BAT (Barthelmy et al. 2005) and XRT (Burrows et al. 2005), during the period bordered by LGRBs 050126 and 110503A inclusively, using the pulse, and afterglow model procedure described in Willingale et al. (2010); a description of which is summarised in Section 2.1. Our definition as to which class, long or short, a GRB belongs to is based solely on the $T_{90}>2 s$ descriminator found by Kouveliotou et al. (1993) and as such may contain some bursts which exhibit the spectral characteristics of short GRBs whilst residing solidly in the LGRB duration regime: so called "extended emission" (EE) bursts

\footnotetext{
2 Many early-time X-ray flares have an observable Gamma-ray counterpart in the BAT however the majority of late-time X-ray flares would have Gamma-ray fluxes well below the BAT detection limit.
} 
(e.g. Norris \& Bonnell (2006), Gompertz, O’Brien \& Wynn (2014)). EE GRBs are a small, and contentious subsample of our GRB dataset and may contribute a small bias in our LGRB formation rates due to the propensity of SGRBs to be detected at low redshifts (D'Avanzo et al. 2014); this is however mitigated to a large extent by the overall number of fitted pulses and as such is not of significant concern.

The following criteria were used to determine the suitability of each GRB for pulse fitting, with the bracketed figures denoting the number of GRBs rejected by the criteria:

- the GRB has an observed redshift and a $T_{90}>2 s(13$ GRBs rejected);

- sufficient statistics in the BAT lightcurve to define at least 1 pulse profile (29 GRBs rejected);

- a BAT lightcurve in which pulses are reasonably well defined (3 GRBs rejected);

- early data from the XRT so that the decay of the pulses is well constrained (22 GRBs rejected); and

- XRT data which provide good definition of X-ray flares, avoiding flares for which profiles are incomplete or broken by orbit gaps etc.. (22 GRBs rejected)

We evaluate any redshift bias that the various rejection criteria may accidentally introduce into our LGRB dataset by computing the 2-sample Anderson-Darling (AD) statistic (Anderson \& Darling 1952; T. W. Anderson 1954; Darling 1957) ${ }^{3}$ on the redshift distributions of accepted GRBs and of GRBs which failed the rejection criteria, under the null hypothesis that both are drawn from the same population. As we are calculating the likelihood of two distributions being drawn from the same parent distribution, no assumptions are required for the shape of the parent; this is not the case were we calculating a one-sample test. We utilise the $\mathrm{k}$-sample Anderson-Darling test codified in the SciPy stats package (Jones, Oliphant \& Peterson 2001), which is based on the work by Scholz \& Stephens (1987). The critical significance values are modelled as a third order polynomial, and interpolated over a percentile grid of $[0.75,0.90,0.95$, $0.975,0.99]$; outside of this range, the $P$-values are extrapolated and, as such, come with large uncertainties the further away one gets. We therefore quote the calculated AD statistic and the appropriate significance level to which the null hypothesis may be rejected.

We can reject the null hypothesis that the redshift distribution of GRBs with a $T_{90}<2 \mathrm{~s}$ is drawn from the same distribution as LGRBs, as the AD statistic of 11.377 corresponds to a significance level of $P>0.99$; this result is entirely expected given our understanding of SGRBs and LGRBs. We also find that we can accept the null hypothesis for both the rejection criteria of early XRT data, and complete XRT flares, having been drawn from the same parent population as our sample of LGRBs: the AD stats are 0.825 , and -0.875 respectively, which corresponds to $P$-values of $P=0.85$ and $P \ll 0.75^{4}$. The criteria for a minimum of one pulse in the BAT, and a well defined BAT pulse are,

3 The Anderson-Darling test statistic is a modified KolmogorovSmirnov test statistic, and is preferred due to its greater sensitivity to differences in the tails of distributions, and its ability to sence differences between very large datasets.

4 The threshold for rejection is often given at the arbitrary $P$ value of $>0.95$. like the $T_{90}$ criterion, both rejected with $P$-values of $>0.99$. It is highly likely, however, that short GRBs have a poorly observed BAT regime, as the hardness of short GRBs does not lend well to detection by the relatively soft BAT passbands. Calculating a 3 -sample AD statistic shows that the null hypothesis that GRBs which fail these three criteria are drawn from the same population can be accepted, with an AD statistic of -0.079 corresponding to a $P$-value of $\ll 0.75$; this suggests that these rejected GRBs are indeed most likely short, and by excluding them we do no insert any significant bias to our LGRB dataset.

In summary, out of 187 LGRBs with associated redshifts covering the 76 month period from GRB 050126 to GRB 110503A with $T_{90}>2 s, 118$ GRB lightcurves were deemed suitable and fitted with 607 pulses: a completeness of $\sim 63 \%$. As a comparison study, Salvaterra et al. (2012) utilised GRBs spanning an almost identical time period as our own and, after applying their selection criteria, drew a population of $58 \mathrm{LGRBs}$ of which 52 have measured redshifts: a completeness of $\sim 39 \%^{5}$.

\subsection{The Pulse Model}

A photon, emitted from the source when a shell is ejected from it, arrives in the observer frame at time $T_{e j}$ which can be thought of as the observed ejection time of the shell. The initial radial time, $T_{0}$ is assumed such that the time at which the first photons emitted from the emission region at radius $R=R_{0}$ reaches the observer at $T=T_{e j}+T_{0}$. Likewise the final radial time, $T_{f}$, emitted from the emission region at $R=R_{f}$ reaches the observer at time $T=T_{e j}+T_{f}$. For compactness of equations we also define two normalised times:

$$
\bar{T} \equiv \frac{T-T_{e j}}{T_{f}} ; \quad \bar{T}_{f} \equiv \frac{T_{0}}{T_{f}} .
$$

Integrating the comoving luminosity over the equal arrival time surface (EATS) in combination with the model spectrum, $B(q)$ (see Equation 4), allows us to derive the flux in terms of number of photons, $N$ per unit energy, $E$, area $A$ and time $T$ :

$$
\frac{d N}{d E d A d T}\left(E, T \geqslant T_{0}+T_{e j}\right)=P\left(\bar{T}, \bar{T}_{f}\right) B(q) ;
$$

the pulse profile, $P\left(\bar{T}, \bar{T}_{f}\right)$, is given by:

$$
P\left(\bar{T}, \bar{T}_{f}\right)=P_{\text {norm }} \bar{T}^{-1}\left[\left(\min (\bar{T}, 1)^{a+2}-\bar{T}_{f}^{a+2}\right)\right]
$$

where the pulse profile includes a normalisation parameter, $P_{\text {norm }}=\left(1-\bar{T}_{f}{ }^{a+2}\right)^{-1}$ such that the value of $P\left(\bar{T}, \bar{T}_{f}\right)$ is 1 at $T=T_{e j}+T_{f}$. The section in square brackets in Equation 3 models the rise in the pulse, in this case controlled

5 The Salvaterra et al. (2012) completeness is derived from the 132 available redshifts that were available at the time of that paper's writing. Whilst the majority of GRB redshifts are released within a few days of initial observation, some are derived, or updated, only after extended follow up observations months, or years, after the initial burst; in all cases we endeavour to obtain the most up to date redshift information available. 


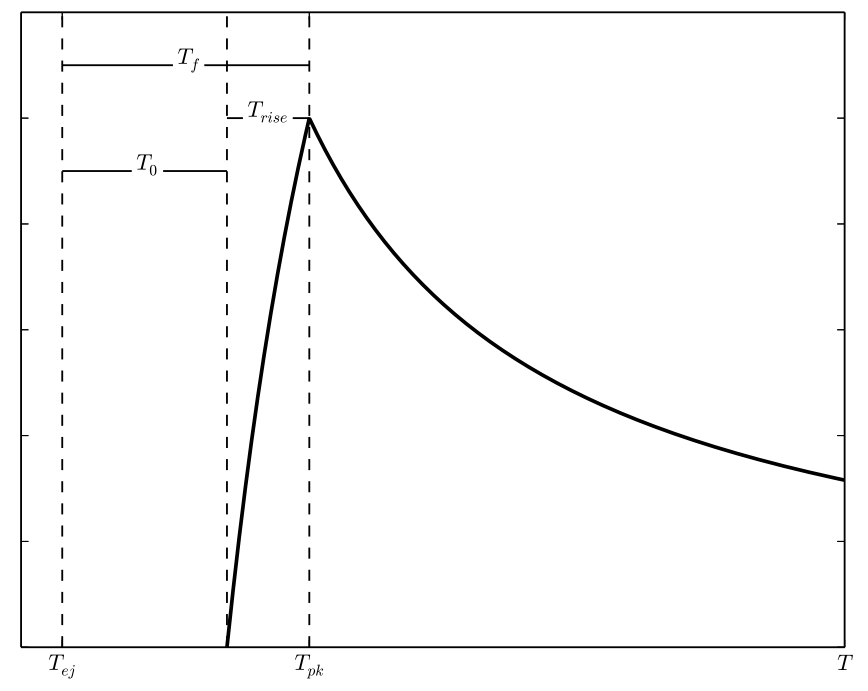

Figure 1. A schematic for the pulse profile, $P\left(\bar{T}, \bar{T}_{f}\right)$, showing the characteristic timescales $T_{f}, T_{0}$, and $T_{\text {rise }}$; and the times $T_{e j}$ and $T_{p k}$.

by the temporal index $a$ and the timescales $\bar{T}$ and $\bar{T}_{f}$. A schematic of the pulse is shown in Figure 1 with characteristic timescales denoted.

Whilst the temporal characteritics of GRBs display remarkable variation, their spectral profiles are far less varied. The spectra of GRB pulses during the prompt phase are distinctly non-thermal; as such, we model each individual pulse by a Band function, given by Equation 4:

$$
B(q)=B_{n} \begin{cases}q^{b_{1}-1} e^{-q} & q \leqslant b_{1}-b_{2} \\ q^{b_{2}-1}\left(b_{1}-b_{2}\right)^{b_{1}-b_{2}} e^{-\left(b_{1}-b_{2}\right)} & q>b_{1}-b_{2}\end{cases}
$$

where $b_{1}$ and $b_{2}$ are the low and high energy spectral indexes respectively, $B_{n}$ is the normalisation parameter, and $q=\left(E / E_{f}\right) \bar{T}=E / E_{c}$. The cutoff energy at time $T_{\text {peak }}=$ $T_{e j}+T_{f}$, denoted as $E_{f}$, coincides with the maximum emission from the pulse where the spectral profile is best constrained. The hard-to-soft evolution seen in GRB pulses are modelled through an evolving characteristic energy, $E_{c}$, such that $E_{c}(t)=E_{c}\left(T-T_{e j}\right)=E_{f}(\bar{T})^{-1}$, where the strength of the spectral evolution is a result of assuming synchrotron dominated emission in the fast-cooling regime (see Willingale et al. 2010 for derivations). Whilst there is, in some rare cases, evidence of an underlying and statistically significant thermal component within the spectra of a few GRBs (see Guiriec et al. 2011 for example), we feel that incorporating an additional thermal component would be an exercise in diminishing returns.

\section{$2.2 \quad$ Fitted parameters}

The time of the peak with respect to the trigger, $T_{p k}$, was initially set by eye and then allowed to float to find the best value. In the final fits $T_{p k}$ was then fixed at the best value found whilst in all cases the characteristic time $T_{f}$ was allowed to float. Instead of fitting the rise time directly it was expressed as a fraction of $T_{f}, T_{\text {rise }}=f_{r} T_{f}$, and it is this fraction which was fitted with the constraint of $0<f_{r}<1$. Using this fraction provides a simple way to avoid unphysical rise time values not allowed by the model. In many bursts significant emission is seen before the trigger and in some bursts the first peak may have a large negative $T_{p k}$ value. On completion of the final fit we redefined the zero time as the start of the emission in the model given by the start of the rise of the first pulse, $T_{z e r o}=T_{p k}(1)-T_{\text {rise }}(1)$. We then offset the peak times of all the pulses in the burst to $T_{\text {peak }}=T_{p k}-T_{z e r o}$ so this represents the time since the start of the burst for each pulse.

For all pulses the low-energy index of the Band function was fit whilst the difference between the low and high spectral indicies was fixed at $b-d=10$. This effectively reduces the Band function to a simpler power law with an exponential cut-off. For a few pulses the count rate in the higher energy channels was effectively zero and the spectral index was very poorly determined. In such cases the lower spectral index was therefore constrained to $b>-2.5$. Because of the relatively soft energy bandwidth of the Swift BAT, and the signal to noise of the measured light-curves, a powerlaw with exponential cutoff produces comparable quality of fits to Band functions without being so computationally demanding.

For the majority of GRB pulses the cut-off energy of the Band function lies outside the passband of the BAT; in such cases we fix the cutoff energy of the Band function at $T_{\text {peak }}$ at $E_{f z}=500 \mathrm{keV}$ in the source frame of the burst, corresponding to $E_{f}=500 /(z+1) \mathrm{keV}$ in the observer frame, similar to the fixed cutoff energies utilised by other studies, e.g. Firmani et al. 2004; Natarajan et al. 2005. For some pulses however, with good statistics and energy coverage (including both the BAT and XRT data), it was possible to constrain $E_{f}$ by the fitting to some other value (usually a lower energy). As joint analysis of the spectra of GRB pulses observed simultaneously by Swift and other satellites such as Fermi, Suzaku, and Konus-Wind are rare and are often based on a few GRBs (see for example Krimm et al. 2009), we cannot directly compare spectral fits on a pulse-by-pulse basis for the majority of our 607 pulses. We instead compare the spectral characteristics of the prompt emission pulses utilised within this paper with the time-averaged spectral parameters observed by other space-based gamma-ray, and X-ray observatories with wider energy passbands than the Swift BAT; out of 118 GRBs, 51 were observed by other missions, totalling 183 prompt-phase pulses.

Although not strictly equivalent, as the totality of the GRB prompt emission is a convolution of many constituent pulses, such a comparison can reveal any significant differences. To this end we define a deviation metric for parameter $P$ such that $\Delta P=\left|P_{\text {Swift }}-P_{\text {other }}\right| / \sigma_{\text {combined }}$ where $\sigma_{\text {combined }}$ is the resulting uncertainty of the two measurements combined in quadrature $\left(\sigma_{\text {combined }}^{2}=\sigma_{\text {Swift }}^{2}+\sigma_{\text {other }}^{2}\right)$, and a $\Delta P<1$ denotes a parameter that is within the combined $1 \sigma$ uncertainties. We find good agreement between our pulse spectral parameters and those of the timeaveraged GRB spectra, with the median deviation in the spectral indexes, and peak energies of $\Delta B_{1}=1.25_{-0.70}^{+1.89}$, and $\Delta E_{\text {peak }}=0.70_{-0.35}^{+0.52}$; where the subscripts/superscripts de- 
note the $25^{t h}$ and $75^{t h}$ percentiles respectively. Such differences in the spectral parameters of our pulses and the timeaveraged GRB prompt emission will produce K-correction factors which may vary significantly, and by extention, produce bolometric rest-frame luminosities that are widely different. We therefore calculate and compare the K-corrections one would derive assuming a power-law with exponential cutoff spectrum for both measurements. We observe a median deviation between the two broadband observations on the scale of $\Delta K_{\text {corr }}=0.28_{-0.15}^{+0.24}$; we conclude therefore that the effect of introducing a fixed cutoff energy in the spectra of our pulses is negligible.

In general the pulse profiles are well matched by the model but Figure 2 illustrates typical deficiencies in the fitting (see Willingale et al. 2010 for further discussion on the various fitting pitfalls). For GRB 060206 the pulse decays more rapidly in the hard band than predicted and there are minor excursions away from the model over several of the pulses in GRB 070508. For many bursts there are a couple of data points in the harder bands which form a spike which is not fitted by the model profile. A few points in the decay of the afterglow in GRB 070508 are well above the model in the softest band and the very late points of the afterglow in GRB 060206 are poorly fit. In these fits such errors can't be accomodated for by the addition of more pulses and subsequently contribute to some of the larger $\chi^{2}$ values obtained. Despite these issues we tried to fit every pulse-like feature in all the lightcurves and the combination of the pulse model plus afterglow accounts, in most cases, for all the emission detected from all the bursts. ${ }^{6}$

\section{MODELLING THE GRB LUMINOSITY FUNCTION}

We note that the nomenclature of "luminosity function" in reference to GRBs refers specifically to the GRB luminosity probability density function $(\mathrm{PDF})$; to obtain what is in general analogous to the LFs found in other areas of astrophysics one must convolve the GRB luminosity PDF with the cosmic GRB formation rates. Any subsequent reference to the GRB luminosity function in this paper will follow this convention and refer to the GRB luminosity PDF. Throughout this paper we used the formulation of comoving distance, volume and luminosity distance given by Hogg (1999) utilising the seven-year WMAP cosmological parameters of $H_{0}=71 \mathrm{~km} \mathrm{~s}^{-1} \mathrm{Mpc}^{-1}, \Omega_{m}=0.27, \Omega_{k}=0$ and $\Omega_{\Lambda}=0.73$ (Larson et al. 2011). All errors quoted in this paper are to the $1 \sigma$ confidence interval in line with the majority of GRB LF literature.

Throughout this paper we discuss reproducing the GRB pulse luminosity function though a variety of models which, in some cases, include various sub-models. Type I models invoke a cosmic star-formation rate coupled to a single population of GRB progenitors (Section 3.2); type II models are similar to type I save for the separation of GRB progenitors into low, and high-luminosity populations (Section 3.1.1); whilst type III models are direct fits to GRB formation rates
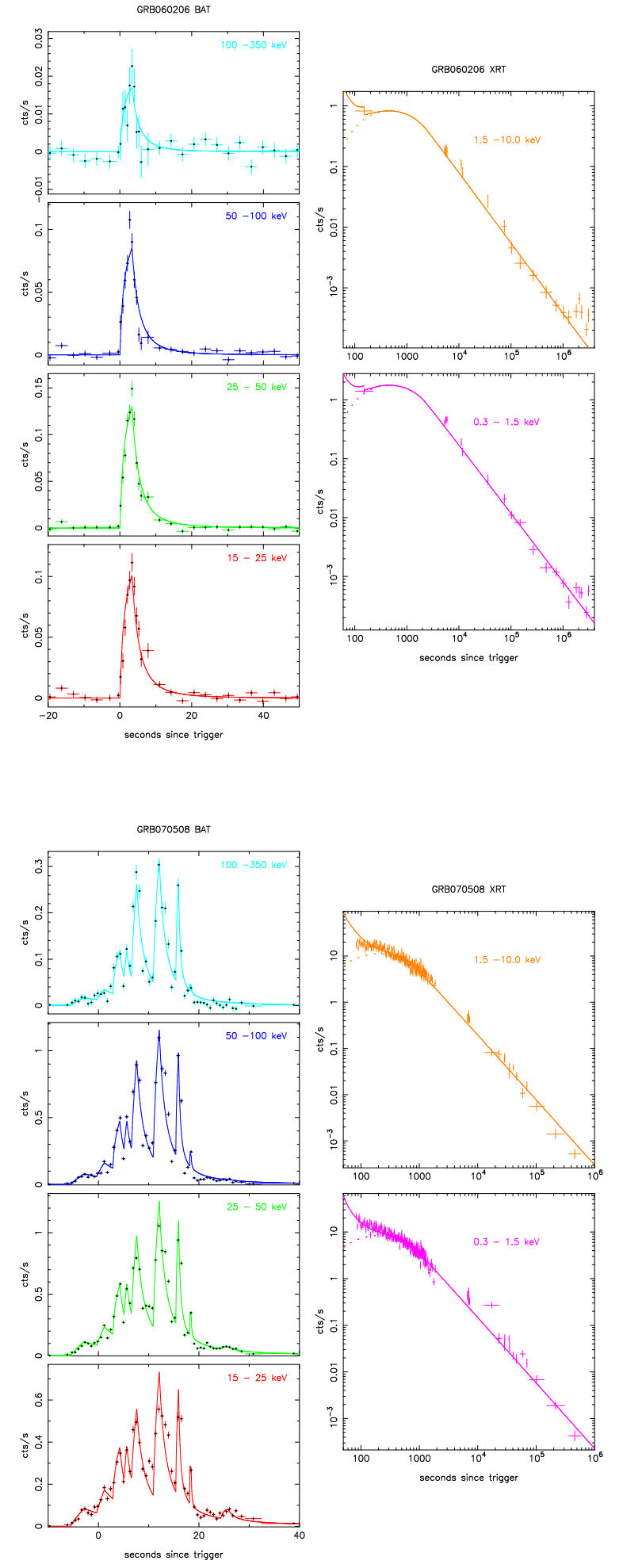

Figure 2. The pulse model fits for GRB 060206 (top) and GRB 070508 (bottom). The left columns corresponds to the BAT passbands of $100-350 \mathrm{keV}$ (cyan), $50-100 \mathrm{keV}$ (blue), $25-50 \mathrm{keV}$ (green), and $15-25 \mathrm{keV}$ (red); the right columns are the XRT passbands of $1.5-10 \mathrm{keV}$ (orange), and $0.3-1.5 \mathrm{keV}$ (magenta).

6 The data used throughout this paper will be available in an upcoming GRB components catalogue paper by the authors. 


\section{A. Amaral-Rogers et al.}

and exclude a-priori assumptions about the nature of GRB progenitors (Section 3.2). Models I, and III are further explored through the inclusion of various extra evolutionary effects (see Section 3.6) and are summarised as:

Type I-1: no evolution in either the break of the pulse LF, $L_{\text {break }}(\delta=0)$, or the GRB formation rate, $K_{G R B}(\gamma=0$ or $\left.Z / Z_{\odot}=\infty\right)$;

Type I-2: evolution of only the GRB formation rate, $K_{G R B}$ $(\gamma \neq 0)$

Type I-3: evolution of only the break, or cutoff, of the luminosity function, $L_{\text {break }}(\delta \neq 0)$;

Type I-4: evolution of the GRB formation rate through the presence of metallicity density evolution $\left(Z / Z_{\odot} \neq \infty\right)$;

Type I-5: both $L_{\text {break }}$ and $K_{G R B}$ are free to evolve $(\delta \&$ $\gamma \neq 0)$.

Type III-1: no evolution in the break of the pulse LF, $L_{\text {break }}(\delta=0)$;

Type III-2: evolution in the break of the pulse LF, $L_{\text {break }}$ $(\delta \neq 0)$;

The observed distribution of pulse bolometric luminosities, $N(L, z)$, by definition spanning the energy band of 1 $10000 \mathrm{KeV}^{7}$, is displayed in Figure 3. Pulses for which the peak only appears in the BAT or XRT lightcurves are shown as circles and stars respectively, whilst pulses observed simulatenously by both instruments are denoted by triangles. The $N(L, z)$ distribution displays a wide range of brightnesses for prompt emission pulses, and late time X-ray flares; and whilst the very brightest of pulses $\left(L_{1-10000 \mathrm{KeV}}>\right.$ $1 \times 10^{53}$ ergs $s^{-1}$ ) are exclusively from the prompt emission, the X-ray flares and prompt emission pulse luminosity distributions are indistinguishable from each other.

The standard procedure for relating the observed distribution of LGRBs to the comoving burst formation rate (see for example Fenimore \& Ramirez-Ruiz (2000); LloydRonning, Fryer \& Ramirez-Ruiz (2002); Salvaterra \& Chincarini (2007); Butler, Bloom \& Poznanski (2010); Salvaterra et al. (2012)) is given by:

$N(L, z) d z d L=\phi(L, z)\left[D(L, z) \frac{\Delta \Omega \Delta T \Psi_{*}(z)}{(z+1)} \frac{d V_{c}(z)}{d z}\right] d z d L$

where the observed distribution of LGRB bursts, $N(L, z)$, is a convolution of the comoving burst formation rate, $\Psi_{*}(z)$, the comoving volume element, $d V_{c}(z) / d z$, a detection probability profile, $D(L, z)$, and the GRB luminosity probability density function, $\phi(L, z)$. The factor of $1 /(z+1)$ corrects for cosmological time dilation whilst $\Delta \Omega$ and $\Delta T$ are the terms correcting for the field of view of the BAT and the total duration our GRB sample covers.

\subsection{Luminosity Function}

The functional forms for LGRB LFs represented in the Swift literature are predominantly that of a broken power-law (sometimes with a smoothed transition between low and

7 The bolometric luminosity of each individual pulse is derived from applying a K-correction to the pulse flux using the spectrum at peak time as a fiducial spectrum.

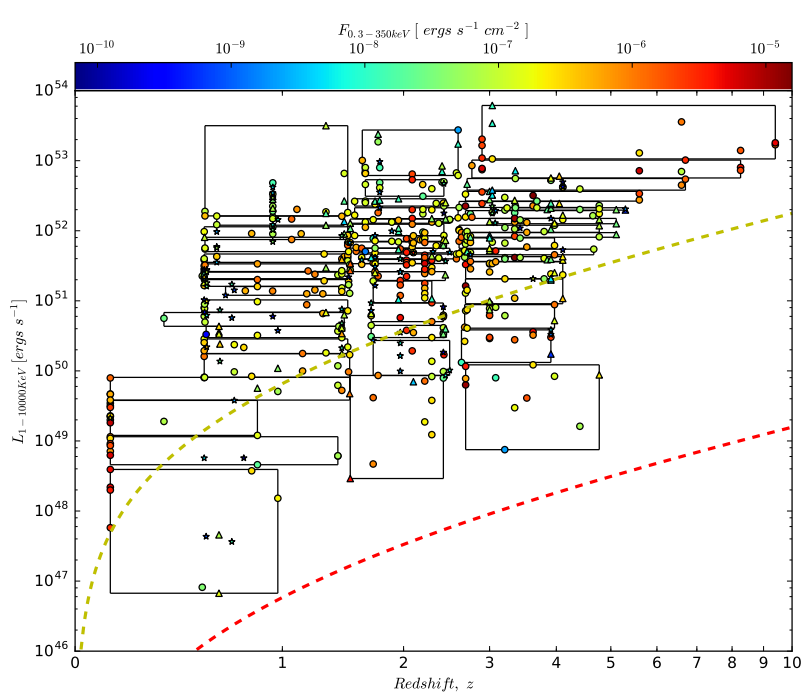

Figure 3. The distribution of peak luminosities as a function of redshift, $N(L, z)$. The circles and stars indicate pulses detected only with the BAT or XRT instruments respectively with triangles denoting pulses detected by both instruments simultaneously. BAT observed pulses are visible below the BAT detection limit as fainter pulses co-added to prior, brighter pulses can be "boosted" into the detection range. Pulse colours denote the integrated peak flux in the observed $0.3-350 \mathrm{keV}$ passband. The overlain boxes show the corresponding $J_{i}$ sets used to determine each bin's limits with an explanation to the derivation of set limits outlined in Section 4.1. The yellow dashed and red dashed lines show the detection threshold of the bolometric luminosity as a function of redshift, with a burst detection threshold in the BAT (15-350 $\mathrm{keV}$ ) of 0.1 photons $\mathrm{cm}^{-2} \mathrm{~s}^{-1}$, equivalent to $8 \times 10^{-9} \mathrm{ergs} \mathrm{cm}^{-2}$ $\mathrm{s}^{-1}$ (Sakamoto et al. 2008) and an approximate XRT (0.3-10 keV) pulse/flare detection limit of $3 \times 10^{-12} \mathrm{ergs} \mathrm{cm}^{-2} \mathrm{~s}^{-1}$. In practice the XRT detection limit depends on the brightness of the afterglow component and the time since trigger so the lower detection threshold varies considerably from burst to burst. Few pulses are seen near the XRT threshold and the detection likelihood of pulses is discussed in Section 3.5. The K-correction to convert the BAT \& XRT flux detection limits to that of bolometric luminosities utilises the average spectral parameters of the Band function derived from our dataset of 607 pulses with $b=-1.57, d=-11.60$ and $E_{f}=183 \mathrm{keV}$.

high luminosity regions) (Lloyd-Ronning, Fryer \& RamirezRuiz 2002; Liang et al. 2007; Butler, Bloom \& Poznanski 2010; Cao et al. 2011; Salvaterra et al. 2012), or a power-law with an exponential cutoff (Salvaterra \& Chincarini 2007; Salvaterra et al. 2009; Cao et al. 2011; Salvaterra et al. 2012). In this paper, to ensure completeness, we utilise both a broken power-law (BPL),

$$
\phi(L, z)=L_{n}\left\{\begin{array}{ll}
\left(\frac{L}{L_{\text {break }}}\right)^{\alpha} & L \leqslant L_{\text {break }} \\
\left(\frac{L}{L_{\text {break }}}\right)^{\beta} & L>L_{\text {break }}
\end{array} ;\right.
$$

and a power-law with exponential cutoff (PLEC),

$$
\phi(L, z)=L_{n}\left(\frac{L}{L_{\text {break }}}\right)^{\alpha} \exp \left[\left(\frac{-L}{L_{\text {break }}}\right)\right] ;
$$

to model our pulse luminosity function. $\alpha$ and $\beta$ (BPL only) are the low \& high luminosity indexes; $L_{\text {break }}$ is the break luminosity; and $L_{n}$ is the normalisation of the LF, which is 
given by the reciprocal of the LF integral. The normalisation factor is sensitive to the limits of integration and can have an effect on the derived efficiency parameter, $K_{G R B}$, up to a factor of 2 . The limits of integration are therefore chosen by various authors depending on the constraints that they place on their data sets, bias controls, or calculation methods ${ }^{8}$; the variation in normalisation is small however when compared to the intrinsic uncertainties in the CSFRD, IMF evolution, metallicity density, etc.. We adopt the faintest, and brightest pulse luminosities as the limits of integration, which in this paper spans $1 \times 10^{46}$ to $1 \times 10^{54} \mathrm{ergs}^{-1}$.

\subsubsection{A Separate Low-Luminosity GRB Population}

Although LGRB studies generally prefer utilising luminosity functions that assume a single population of LGRBs, a small group of LGRBs appear to exist with particularly low luminosities (LL, $L<10^{50}$ ergs $s^{-1}$ ) that are poorly fitted by these single population models (Liang et al. 2007; Virgili, Liang \& Zhang 2009; Qin et al. 2010; Howell \& Coward 2013). Typically these LL LGRBs are assumed to trace the same progenitor models as those of higher luminosity LGRBs whilst convolved to a separate luminosity function. Such luminosity functions produce markedly differing normalisation rates for the two types of LGRBs; the local formation rates of LL LGRBs are suggested to be several orders of magitude greater than those of more luminous LGRBs.

With the incorporation of bright prompt emission pulses, and late time, faint X-ray flares, 72 of 607 pulses fall into the luminosity regime typically associated with LL LGRBs. In this paper we evaluate the performance of bimodal LF models (denoted as type II models) compared to single population LGRB models (type I models). Following a similar procedures set out by Liang et al. (2007), we produce a bimodal luminosity function by combining two luminosity functions, $\phi_{L L}(L)$ and $\phi_{H L}(L)$ such that:

$$
\phi(L)=\phi_{L L}(L) K_{L L-G R B}+\phi_{H L}(L) K_{H L-G R B},
$$

where the LGRB formation rate efficiencies, $K_{L L-G R B}$ and $K_{H L-G R B}$ are included in the LF to allow for different formation efficiencies of the two GRB types, and are analogous to the $\rho_{0}^{L L}$ and $\rho_{0}^{H L}$ parameters found in Liang et al. (2007). Both $\phi_{L L}(L)$ and $\phi_{H L}(L)$ follow the same shape as Equations 6 and 7 and each population is fitted separately to ensure that the LL and HL parameters are independent of each other. Normalisation limits for the bimodal LFs, as that of the single population model, are set at $1 \times 10^{46}$ to $1 \times 10^{54}$ $\operatorname{ergs} s^{-1}$.

\footnotetext{
8 The brightest subsection of low- $z$ GRBs are often utilised as the subsample avoids Malmquist bias, and is less succeptible to other intrinsic biases such as redshift detectability, and uncertainty in the CSFRD at high- $z$ (Cao et al. 2011); utilising the least/most luminous pulses (Firmani et al. 2006; Salvaterra et al. 2012), or integrating over infinity, especially for PLEC LF models (Campisi, Li \& Jakobsson 2010), is also common.
}

\subsection{LGRB Co-moving Pulse Rate}

We model the comoving burst rate, $\Psi_{*}(z)$, or more specifically the comoving pulse formation rate (pulses $y r^{-1}$ $M p c^{-3}$ ) using two diametrically opposed models:

$$
\Psi_{*}(z)=K_{\text {pulse }}\left\{\begin{array}{ll}
K_{G R B} \psi_{*}(z) \iota(z) F(z) & \text { Type I } \\
\psi_{G R B}(z) & \text { Type III }
\end{array} .\right.
$$

Type I models assume a functional form for the cosmic star formation rate density (CSFRD), $\psi_{*}(z)\left(M_{\odot} y r^{-1}\right.$ $M p c^{-3}$ ), and couple to: an evolving fraction of high-mass stars that are capable of forming GRBs at at given redshift, $F(z)$; an additional rate density evolution parameter, $\iota(z)$, capable of boosting GRB formation rates above CSFRD levels, and conversion factors $K_{\text {pulse }}$, and $K_{G R B}$ which describe the average number of pulses per GRB, and the number of GRBs formed per solar mass of stars respectively. Included amongst the type I models is a non-evolving GRB luminosity function derived when $\iota(z)$ is constant, and the break luminosity index, $\delta=0$. Type III models are a common alternative to type I models where direct fitting of a simple functional form to $\Psi_{*}(z)$, in this paper taken to be a triple broken power-law, allows for ease of comparison between cosmic star formation rate density models without the need for refitting of GRB luminosity functions.

All the parameters used in modelling the comoving pulse formation rate are functions of redshift with the exception of $K_{\text {pulse }}$ : the number of pulses per GRB shows no correlation with redshift; having removed the effect of the BAT rest-frame duration, $T_{90} /(z+1)$, we derive a Spearmann's partial rank correlation coefficient of $\rho_{s}=-0.045$, implying that $K_{\text {pulse }}$ is redshift-independent.

\subsection{Cosmic Star Formation Rate Density}

The comoving burst formation rate is dependant on the properties of the central engines that power GRBs; for LGRBs the preferred mechanism is that of a collapsar: massive stars that undergo catastrophic core collapse into blackholes (Woosley 1993; Paczyński 1998; MacFadyen \& Woosley 1999), favoured because of the observed association with Type Ib/c supernovae (Galama et al. 1998; Stanek et al. 2003) with Wolf-Rayet stars the favoured progenitor type. With their high mass $\left(M>25 M_{\odot}\right)$, and subsequently short main-sequence lifespans, Wolf-Rayet stars closely trace the local star formation rate; as such, for type I/II models, we take the Cole (Cole et al. 2001) functional form for the CSFRD:

$$
\psi_{*}(z)=\frac{\left(a_{1}+a_{2} z\right) h}{1+\left(z / a_{3}\right)^{a_{4}}},
$$

in units of $M_{\odot} y r^{-1} M p c^{-3}$; and use the best fit parameters: $a_{1}=0.0389, a_{2}=0.0545, a_{3}=2.973$, and $a_{4}=3.655$ derived by Kobayashi, Inoue \& Inoue (2013). These values are based on corrections to the work by Hopkins \& Beacom (2006) where overestimations in the CSFRD were found to have arisen due to uncertainties in the correction for dustobscuration and the conversion from UV luminosity to intrinsic star formation rates. These coefficients produce a cosmic star formation rate that has an almost flat profile to a 
redshift of $z=2$ and approximately an order of magnitude greater formation rate at $z=0$ than that produced from using Hopkins and Beacom's fitted parameters.

\subsection{The Cosmic IMF}

A contributing second-order effect from an evolving population of high-mass stars is considered by some authors either explicitly in the modelling of derived GRB luminosity functions (Lloyd-Ronning, Fryer \& Ramirez-Ruiz 2002) or as an explanation to the observed evolution in luminosity or rate parameters (Kistler et al. 2008; Cao et al. 2011). The CSFRD is, by definition, the total star formation rate at a given redshift and, for completeness, in this paper we explicitly convert the CSFRD to a formation rate density of stars capable of undergoing catastrophic core collapse and forming GRBs (i.e. with mass greater than $25 M_{\odot}$ ) by deriving the fractional mass of stars greater than a "GRB ignition mass", $F(z)$, given by $F(z)=\int_{25 M_{\odot}}^{120 M_{\odot}} M \Phi(M, z) d M / \int_{0.01 M_{\odot}}^{120 M_{\odot}} M \Phi(M, z) d M$. In our derivation of the fraction of high-mass stars we assume an IMF, $\Phi(M, z)$, which is top-heavy at high redshift as logically in the metal-poor early universe the Eddington limit, and subsequently the population of high mass stars, was much greater than more recent epochs. Studies into extragalactic star formation history indicates an evolving IMF (Davé 2008; van Dokkum 2008; Wilkins, Trentham \& Hopkins 2008) up to $z \sim 2$ and as such we adopt the redshiftdependent IMF model of Davé (2008) where the IMF takes the form of a broken power-law (Kroupa 2001):

$$
\Phi(M, z)= \begin{cases}\left(\frac{M}{\hat{M}}\right)^{-0.3} & M \leqslant \hat{M} \\ \left(\frac{M}{\hat{M}}\right)^{-1.3} & M>\hat{M}\end{cases}
$$

with the characteristic break mass evolving with redshift: $\hat{M}=0.5(z+1)^{2} M_{\odot}$, which we naively extrapolate up to $z=10$. The effect of the evolving IMF on the distribution of stellar masses is subtle; in the current epoch, approx. 9.6\% of all stellar mass formed per year is locked up within stars of $M>25 M_{\odot}$, increasing to approx. $62.5 \%$ at $z=10$.

\subsection{Swift Detection Likelihood}

It is common, in previous studies of the Swift GRB luminosity function, where only the defining pulse luminosities (i.e. the brightest) were utilised, to set the likelihood of detection by the BAT within its field of view to be at unity. In deriving a GRB pulse luminosity function incorporating data from the XRT we include pulses up to three orders of magnitude less luminous than the detection threshold of the BAT. We produce a model of the Swift detection profile, $D(L, z)$, assuming total detection likelihood above the BAT detection threshold which scales to zero at an effective XRT detection threshold of $3 \times 10^{-12}$ ergs $\mathrm{s}^{-1} \mathrm{~cm}^{-2}$ as a power-law of index $\sim-1 / 3$. This is, of course, a naive model of Swift's detection profile: each pulse is treated as an individual event and assuming unity down to the XRT detection threshold would be inappropriate; each pulse detected by the XRT was because of BAT detection and the XRT detection threshold varies considerably from burst to

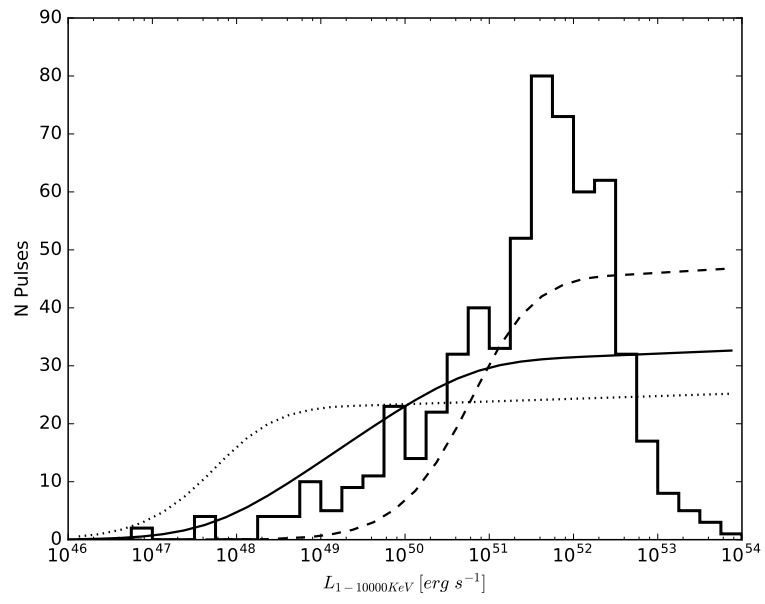

Figure 4. The observed distribution of pulse luminosities for a type I model with rate evolution of $\gamma=0$ where the luminosity function is assumed to be constant. The solid curve is the expected distribution resulting from integrating Equation 5 whilst assuming a detection profile, $D(L, z)$, that varies from unity at the BAT threshold down to non-detection at the approximate XRT threshold. The dotted curve is the expected distribution assuming $D(L, z)$ is unity down to the XRT sensitivity limit and the dashed curve arises when setting the BAT detection threshold as the lower limit of detection.

burst depending on the brightness of the afterglow component, and the time between XRT detection and BAT trigger; furthermore as there is often significant overlap between pulses, fainter pulses may be seen when an earlier, significantly brighter pulse is present. Modelling the combinded detection profile of Swift is highly complicated and, as such, the results are somewhat subjective. Our detection profile convolved to the CSFRD, metallicity density, and constant $\phi(L, z)$, produces a distribution of pulses that closely traces the observed distribution up to approximately $10^{51}$ ergs $s^{-1}$ (solid line, Figure 4). Setting the detection profile to unity above either the XRT or BAT detection thresholds produces the dotted and dashed distributions which tends to overestimate the population of low luminosity pulses $(>\mathrm{XRT}=$ unity) or underestimates the population of sub-peak luminosities $(\angle \mathrm{BAT}=0)$ requiring, respectively, a luminosity function that is more positively or negatively tilted to compensate.

\subsection{Redshift Evolution Models}

For a type I GRB LF model, the basic method of taking a CSFRD convolved to a luminosity function, detection profile, and cosmological volume element produces a distribution of LGRBs that under-represents the observed highredshift, high-luminosity population. The solution is to provide an extra evolutionary effect in the modelling and allow it to float when fitting the model parameters. In this paper we look at three of the most common evolutionary effects: evolution of the break, or cutoff, of the luminosity function; a metallicity density evolution such that LGRBs trace low metallicity star forming regions; and a more generic rate density evolution on top of the CSFRD as solutions to differ- 
ences between the observered and type I pulse distribution functions.

\subsubsection{Break Luminosity}

Evolution in the break, or cutoff, luminosity is of the form $L_{\text {break }}(z)=L_{0}(z+1)^{\delta}$, where $L_{0}$ is the break in the LF at $z=0$ and $\delta$ is the index of LF evolution (Lloyd-Ronning, Fryer \& Ramirez-Ruiz 2002; Firmani et al. 2004; Yonetoku et al. 2004; Kocevski \& Liang 2006; Salvaterra \& Chincarini 2007; Salvaterra et al. 2009; Campisi, Li \& Jakobsson 2010; Virgili et al. 2011; Salvaterra et al. 2012; Petrosian, Kitanidis \& Kocevski 2015; Yu et al. 2015). This has the further effect that the normalisation parameter, $L_{n}$, becomes $L_{n}(z)$. In this paper the break luminosity evolution can be applied to both the type I and type III LGRB pulse formation rate models. In principle, luminosity break evolution can be incorporated into type II models such that either one, or both, GRB populations see their own luminosity evolution. Given the large number of free parameters, and the small population of low-luminosity pulses, however, we believe that we do not yet have the statistics to draw meaningful conclusions from such a model.

\subsubsection{Metallicity Density}

Extreme mass-loss through stellar winds, a characteristic of high-mass stars, will prevent the formation of a GRB; if, however, the progenitor has low metallicity $\left(Z<0.1 Z_{\odot}\right)$ then the mass-loss rate is severely dampened and a GRB is able to form (Fryer, Woosley \& Hartmann 1999; Mészáros 2006). LGRB progenitors should therefore preferentially form in low-metallicity galaxies at any given redshift. A model of fractional mass densities belonging to metallicities below metallicity $Z$ at redshift $z, \Sigma(z)$ has been derived by Langer \& Norman (2006) from the Schechter distribution function of galaxy masses and the mass-metallicity relationship determined from SDSS surveys. The functional form of $\Sigma(z)$ is given by:

$$
\Sigma(z)=\frac{\hat{\gamma}\left[0.84,\left(Z / Z_{\odot}\right)^{2} 10^{0.3 z}\right]}{\Gamma(0.84)}
$$

where $\hat{\gamma}$ and $\Gamma$ are the lower incomplete and complete gamma functions respectively. The metallicity density will always boost high-redshift GRB formation rates, with the metallicity threshold determining how rapidly this rate increases; a higher metallicity threshold will produce a smaller increase in GRB formation with redshift, tending towards no evolution when $Z / Z_{\odot} \rightarrow \infty$ (Salvaterra \& Chincarini 2007; Qin et al. 2010; Virgili et al. 2011; Salvaterra et al. 2012).

\subsubsection{Rate Density}

Metallicity density evolution acts as a physical explaination to observed evolution in GRB formation rates, however the formulation of the model relies on no scatter in the mass-metallicity relationship, and no redshift evolution in the faint end of the Schechter galaxy mass function and the rate of which the average galactic metallicity evolves. One may instead use a simple $(z+1)^{\gamma}$ factor to produce the same effect as metallicity density evolution with the advantage that rate density also allows for a dampening of GRB formation rates at high- $z$, something that is impossible for the formulation of metallicity density to achieve (Kocevski \& Liang 2006; Kistler et al. 2008; Salvaterra et al. 2009; Qin et al. 2010; Cao et al. 2011; Virgili et al. 2011; Robertson \& Ellis 2012; Salvaterra et al. 2012; Petrosian, Kitanidis \& Kocevski 2015). This factor is however purely empirical, which frustrates interpretations of the results. Both the metallicity density and rate density evolution are incorporated into the type I GRB formation rate model, $\Psi_{*}(z)$, through the $\iota(z)$ term in Equation 9, either singularly or in combination with each other (Qin et al. 2010).

\subsubsection{Combined Break Luminosity 8 Rate Density}

Evolution either in rate density, or break luminosity has been utilised as a solution to discrepencies between theoretical, and observed LGRB luminosity functions. Little study has however been made on the performance of more complex evolutionary models involving evolution in both rate and break luminosity. In this paper we evaluate the performance of a type I combined rate/break evolutionary model and compare this model's performance with the more common univariate type I evolutionary models.

\section{THE GRB PULSE LUMINOSITY FUNCTION}

\subsection{The MCMC Simulation}

We bin the observed distribution, $N(L, z)$, by splitting the 607 GRB pulses into equipopulous redshift bins: $0.125<$ $z \leqslant 1.505,1.51<z \leqslant 2.6$, and $2.612<z \leqslant 9.4$; we furthermore bin over luminosity to improve statistics at the high and low luminosity tails of the GRB pulse distribution such that the $i$ th bin is the associated set $J_{i} \equiv\left\{j \mid L_{i}^{\text {min }}<L<\right.$ $\left.L_{i}^{\max }, z_{i}^{\min }<z<z_{i}^{\max }\right\}$, with $n\left(J_{i}\right)$ as the total number of pulses in $J_{i}$, set at a minimum of 11 pulses: a tradeoff between maximising the total number of bins, and reducing the fractional Poissonian error component of each bin. The lower and upper redshift and luminosity limits of each bin are subsequently trimmed to remove excess "padding" of empty data space with the resulting bins shown in Figure 3. For a non-trivial model with parameters, $\hat{\theta}$, a Gaussian minus log-likelihood function can be constructed using methods outlined by D'Agostini (2005), giving:

$$
\begin{aligned}
&-\log \left(\mathcal{L}\left[\hat{\theta} \mid n\left(J_{i}\right)\right]\right)=\sum_{i=1}^{N} \frac{\log \left(2 \pi \sigma_{i}^{2}\right)}{2} \\
&+\sum_{i=1}^{N} \frac{\left[n\left(J_{i}\right)-\int_{L_{i}^{m i n}}^{L_{i}^{\max }} \int_{z_{i}^{\text {min }}}^{z_{\text {max }}^{\text {max }}} \eta(L, z ; \hat{\theta}) d L d z\right]^{2}}{2 \sigma_{i}^{2}}
\end{aligned}
$$

where $\eta(L, z ; \hat{\theta})$ is equivalent to the R.H.S of Equation 5 and the associated squared error of the $i$ th bin is given by $\sigma_{i}^{2}=\sigma_{n\left(J_{i}\right)}^{2}+\sigma_{L_{i}}^{2}+\sigma_{z_{i}}^{2}$. The error in $n\left(J_{i}\right), \sigma_{n\left(J_{i}\right)}$ is naively taken as the standard deviation of a Poissonian distribution with mean, $n\left(J_{i}\right)$, giving $\sigma_{n\left(J_{i}\right)}=\sqrt{n\left(J_{i}\right)}$. The errors, $\sigma_{L_{i}}$, and $\sigma_{z_{i}}$ are defined as uncertainties in the limits of integration for each bin. As the bin edges are defined only by 
the minimal/maximal pulse luminosities and redshifts contained theirin, assuming a $10 \%$ uncertainty in the limits of integration gives:

$$
\begin{aligned}
& \sigma_{L_{i}}= \int_{0.9 L_{i}^{\text {min }}}^{1.1 L_{i}^{\max }} \int_{z_{i}^{\text {min }}}^{z_{i}^{\max }} \eta(L, z ; \hat{\theta}) d L d z \\
&-\int_{L_{i}^{\text {min }}}^{L_{i}^{\max }} \int_{z_{i}^{\text {min }}}^{z_{i}^{\max }} \eta(L, z ; \hat{\theta}) d L d z ; \\
& \sigma_{z_{i}}=\int_{L_{i}^{\text {min }}}^{L_{i}^{\max }} \int_{0.9 z_{i}^{\text {min }}}^{1.1 z_{i}^{\max }} \eta(L, z ; \hat{\theta}) d L d z \\
& \quad-\int_{L_{i}^{\text {min }}}^{L_{i}^{\text {max }}} \int_{z_{i}^{\text {min }}}^{z_{i}^{\text {max }}} \eta(L, z ; \hat{\theta}) d L d z .
\end{aligned}
$$

A Metropolis-Hastings Markov Chain Monte Carlo (MCMC) method is preferred for the maximisation of the minus log-likelihood due to the high dimensionality of the fitting, as well as being able to return the confidence regions of all fitted parameters. Assuming uniform priors for the indexes: $\alpha, \beta, \gamma$, and $\delta$; and logarithmic priors for $K_{G R B}$ and $L_{0}$, we run MCMCs with chain lengths of $1 \times 10^{6}$ with typical "burn in" taking around $2 \times 10^{3}$ iterations. To ensure that the MCMC program is finding the global, rather than local, maximum we evaluate the MCMC convergence success by running multiple MCMCs from random starting points and deriving the Gelman \& Rubin (Gelman \& Rubin 1992) potential scale reduction factors (PSRFs); an example for the fully evolving PLEC model, allowing GRB rate and break luminosity evolution, is shown in Figure 5.

For the type I models our results are discussed in Section 5 and tabulated in Table 1; the results derived using the type II bimodal low-luminosity and high-luminosity functions are discussed in Section 6 and displayed in Table 2; and the results for a type III LF independent of formation rate models are discussed in Section 7 and shown in Table 3 . The $\chi_{r}^{2}$ quoted are derived from the 54 bins shown in Figure 3, the associated error of the $i$ th bin, $\sigma_{i}$, and the number of fit parameters of the model. The Akaike weights, $w_{i}\left(A I C_{c}\right)$, derived using the Akaike Information Criterion (AIC) (Akaike 1974), are shown and are a measure of the relative likelihood of each model. Derived from $w_{i}\left(A I C_{c}\right)=\exp \left(-\Delta\left(A I C_{c}\right)_{i} / 2\right) / \Sigma\left[\exp \left(-\Delta\left(A I C_{c}\right)_{i} / 2\right)\right]$ where $\Delta\left(A I C_{c}\right)_{i}=A I C_{c}-\min \left(A I C_{c}\right)$, they can be considered as the probability that model $M_{i}$ is the best amongst all the chosen models and penalises models with larger numbers of free parameters. The $A I C_{c}$ is used rather than the $A I C$ as it contains extra-terms that adjust for the bias that a finite sample size can contain.

\section{THE TYPE I GRB MODELS}

\subsection{No Evolution Model (Type I-1)}

We find that the scenario in which there is no inclusion of evolutionary models: luminosity break, rate density, or metallicity, produces a fit of $\chi_{r}^{2}=[1.81,1.83]$ for the BPL and PLEC models respectively. This model produces a distribution of GRB pulses that underestimates the extrema of

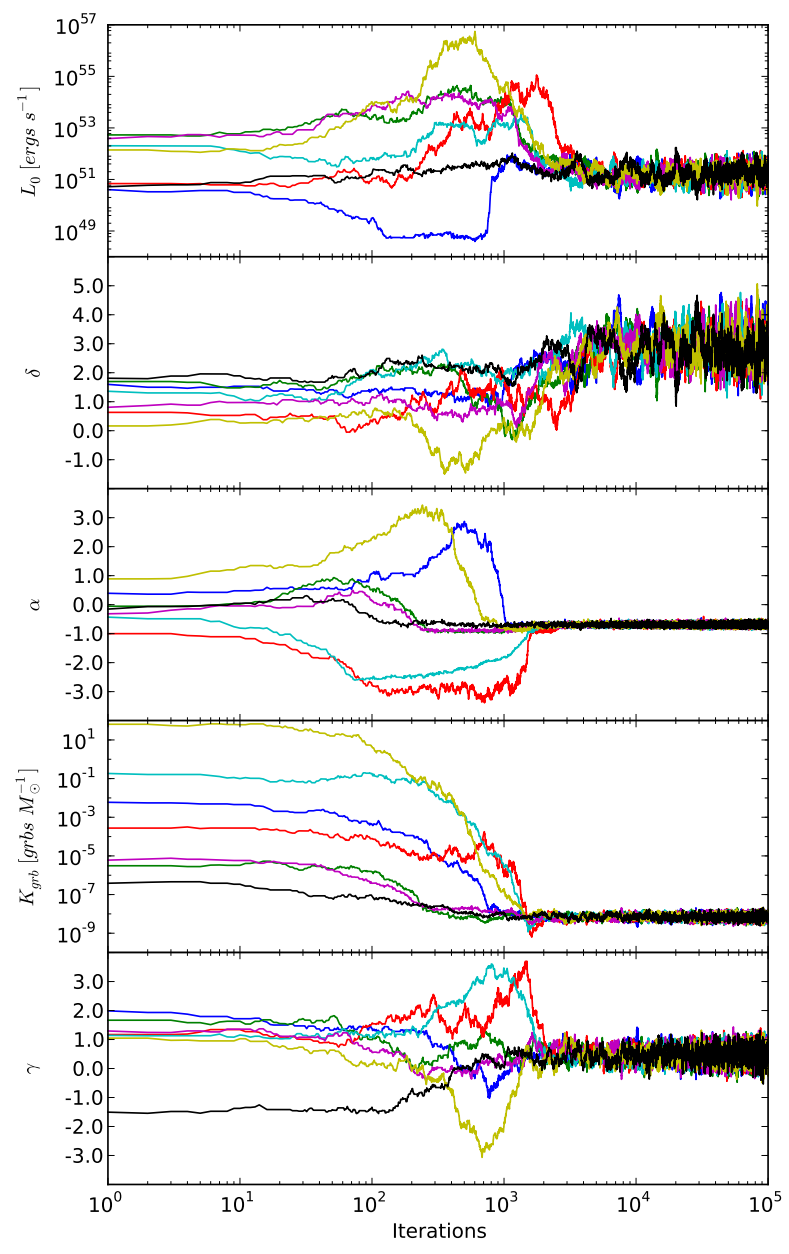

Figure 5. $7 \mathrm{MCMC}$ chains for the type I GRB pulse LF PLEC model convolved with the CSFRD, and the fractional population of high mass stars. The parameters for the break luminosity, $L_{0}$; luminosity break evolution, $\delta$; low-luminosity index, $\alpha$; GRB formation rate, $K_{g r b}$; and GRB formation rate evolution, $\gamma$, were allowed to evolve from random start points and converge to a unique solution typically around $\sim 2 \times 10^{3}$ iterations. Gelman \& Rubin PSRFs statistics for each of the parameters were derived with $\sigma_{P S R F}=1.0051,1.0073,1.0058,1.0022,1.0052$ for $L_{0}, \delta, \alpha$, $K_{g r b}$, and $\gamma$ respectively.

the observed pulse luminosity distribution. The derived normalised Akaike information criterion weights, $w_{i}\left(A I C_{c}\right)$ for the BPL and PLEC LF models are $\sim \times 10^{-7}$, making these models highly unlikely, compared to the fully evolving LF and GRB rate type I-5 models, to minimise the KullbackLeibler discrepancy and as such we can reject this model. This finding is in agreement with single pulse studies utilising the brightest prompt emission pulses (Daigne, Rossi \& Mochkovitch 2006; Salvaterra \& Chincarini 2007; Salvaterra et al. 2009; Qin et al. 2010; Wanderman \& Piran 2010; Virgili et al. 2011; Salvaterra et al. 2012). 


\section{The Pulse Luminosity Function of Swift Gamma-ray Bursts}

\subsection{Rate Density Model (Type I-2)}

The addition of a simple $(z+1)^{\gamma}$ rate evolution produced a best fit to the observed pulse distribution of $\gamma=0.51 \pm 0.30$ for the BPL and PLEC models (top left panel, Figure 6). This shifts the peak of the CSFRD to higher redshifts, boosting the GRB pulse formation rate at high- $z$ whilst reducing low- $z$ formation rates, producing broadly the same deficiencies as the non-evolving type I-1 model with regards to reproducing the observed population of LGRBs at the extrema. A marginal improvement in the fits of $\chi_{r}^{2}=[1.78,1.78]$ is seen and the addition of the extra evolutionary parameter makes this model approximately twice as likely as the non-evolving type I-1 model to produce our observed GRB pulse distribution according to Akaike weighting. This is however still approximately $10^{6}$ times less likely than the fully evolving type I-5 model, making this model highly unlikely and as such we reject it as a solution to the observed evolution in the GRB pulse distribution.

Our derived $\gamma$ values are consistent with those derived in single pulse GRB LF studies, albeit towards the lower end of the distribution $(0.5<\gamma<1.93$, Kistler et al. (2008); Qin et al. (2010); Cao et al. (2011); Virgili et al. (2011); Robertson \& Ellis (2012); Salvaterra et al. (2012); G. Dainotti et al. (2014)). This diversity, in part, reflects the diversity of GRB formation models used, most notably the CSFRD, and the selection methods of suitable GRBs preferred by the authors. Furthermore, excluding the evolving formation rate efficiency of high-mass stars, $F(z)$, which itself produces a weak rate evolution, would result in a greater derived $\gamma$ value as such effects are ignored in other papers. Direct comparisons between studies are difficult given the variation in methods, and data utilised, however the common result is that inclusion of a rate density parameter improves the performance of the fit but is less effective than other evolutionary models (see Salvaterra et al. (2012) for example).

\subsection{Metallicity Density Model (Type I-4)}

Our attempts at fitting metallicity density evolution proved to be unsuccessful, with our MCMC code unable to converge on a unique solution, suggesting strong degeneracy between $Z / Z_{\odot}$ and other fitted parameters. We therefore chose to set six metallicity thresholds and fit our data, covering $0.01<Z / Z_{\odot}<0.6$. We find that degeneracy exists between the metallicity threshold, and all other fitted parameters, with this degeneracy arising from the unique shape of $\Sigma(z)$. The functional form of $\Sigma(z)$ can be crudely considered as a linear rise in $z$ connecting two plateaus at $\Sigma(z) \approx 0$ and $\Sigma(z) \approx 1$. The metallicity density threshold acts to shift $\Sigma(z)$ in $z$, whereby a greater $Z / Z_{\odot}$ shifts the start of the linear rise to lower- $z$. For $Z / Z_{\odot}=0.01$, this shift is strong enough that the majority of $\Sigma(z)$ is at the first plateau, resulting in a significantly higher $K_{G R B}$ to compensate. As $Z / Z_{\odot}$ increases, more of $\Sigma(z)$ occupies the upper plateau and $K_{G R B}$ tends towards values found for type I models excluding metallicity density evolution. Further degeneracy between $Z / Z_{\odot}$ and $L_{0}, \alpha$, and $\beta$ arises when convolving $\Sigma(z)$ to the Swift detection profile, $D(L, z)$. As the detection thresholds of the BAT and XRT effectively bisects the $L-z$ plane, changes to the size of the plateau that $\Sigma(z)$ produces is rotated onto the $L$ dimension by $D(L, z)$, and is counterbalanced by variation of the LF parameters.

Despite the range of metallicity density thresholds fitted, our type I-4 models produces broadly similar quality of fits, with small variations as displayed by the $\chi_{r}^{2}$ and Akaike weights in Table 1 . Across all $Z / Z_{\odot}$ in both the BPL and PLEC we see a general improvement in the quality of fits as compared to both the non-evolving type I-1 model and type I-2 rate density model. The combined Akaike weights make the type I-4 metallicity density model 153 times more likely than the non-evolving type I-1 model and 70 times more likely than the type I- 2 rate density model whilst the evolving type I-3 LF break model is $3.7 \times 10^{3}$ more likely. These values strongly suggests that either the metallicity density evolution is not a suitable explaination to the observed distribution of GRB pulses, or that assumptions made in the derivation of $\Sigma(z)$ are not entirely appropriate. The derivation for the metallicity density evolution by Langer \& Norman (2006) does not, for example, consider scatter in the mass-metallicity relationship, redshift evolution of the faint end of the SGMF, or the rate at which the average galactic metallicity evolves with redshift.

Although the degeneracies of the metallicity density prevents suitable convergence in the metallicity density threshold, our results are broadly similar to studies utilising a GRB's brightest pulse: Salvaterra et al. (2012) finds that metallicity density evolution is more likely than rate density evolution and less likely than evolution in the break to minimise information loss, although to a much less significant degree than we find; Qin et al. (2010) finds that a GRB formation rate that is proportional to both CSFRD and metallicity density (with $Z / Z_{\odot}=0.1$ ) only barely reproduces the $z$ distribution; whilst Virgili et al. (2011) finds such models failed to reproduce observations to enough significance to pass the author's criteria.

\subsection{Break Luminosity Evolution (Type I-3)}

Evolution in the break, or cutoff, of the LF model is the most common explaination to the observed evolution in the GRB distribution. We find that the inclusion of break evolution produces an evolutionary factor $\delta$ of $3.35_{-0.74}^{+0.74}$ and $2.92_{-0.54}^{+0.54}$ for the BPL and PLEC models with corresponding $\chi_{r}^{2}$ values of 1.05 and 1.08. As seen in the top right panel of Figure 6 the evolution in the break acts to boost the GRB pulse distribution at the extrema, significantly improving the fit statistics. Combined Akaike weights, $w_{i}\left(A I C_{C}\right)$ of 0.196 , for the evolving LF break model shows that this model is $3 \times 10^{3}$ times more likely than type I- 4 metallicity density models, and $2.8 \times 10^{5}$ times more likely than type I-2 rate density models to minimise information loss; luminosity evolution in the GRB LF, excluding or including all secondary GRB pulses, is preferred over all over forms of type I univariate evolutionary models (Salvaterra \& Chincarini 2007; Salvaterra et al. 2012).

Our derived values for the LF break evolution parameter are consistent with GRB LF studies that utilise Swift data $(2.1<\delta<3.5$, Yonetoku et al. (2004); Campisi, Li \& Jakobsson (2010); Salvaterra et al. (2012); Petrosian, Kitanidis \& Kocevski (2015); Pescalli et al. (2015); Yu et al. (2015)), whilst studies that incorporate BATSE data display weaker luminosity evolution $(1.0<\delta<2.0$. Lloyd-Ronning, 


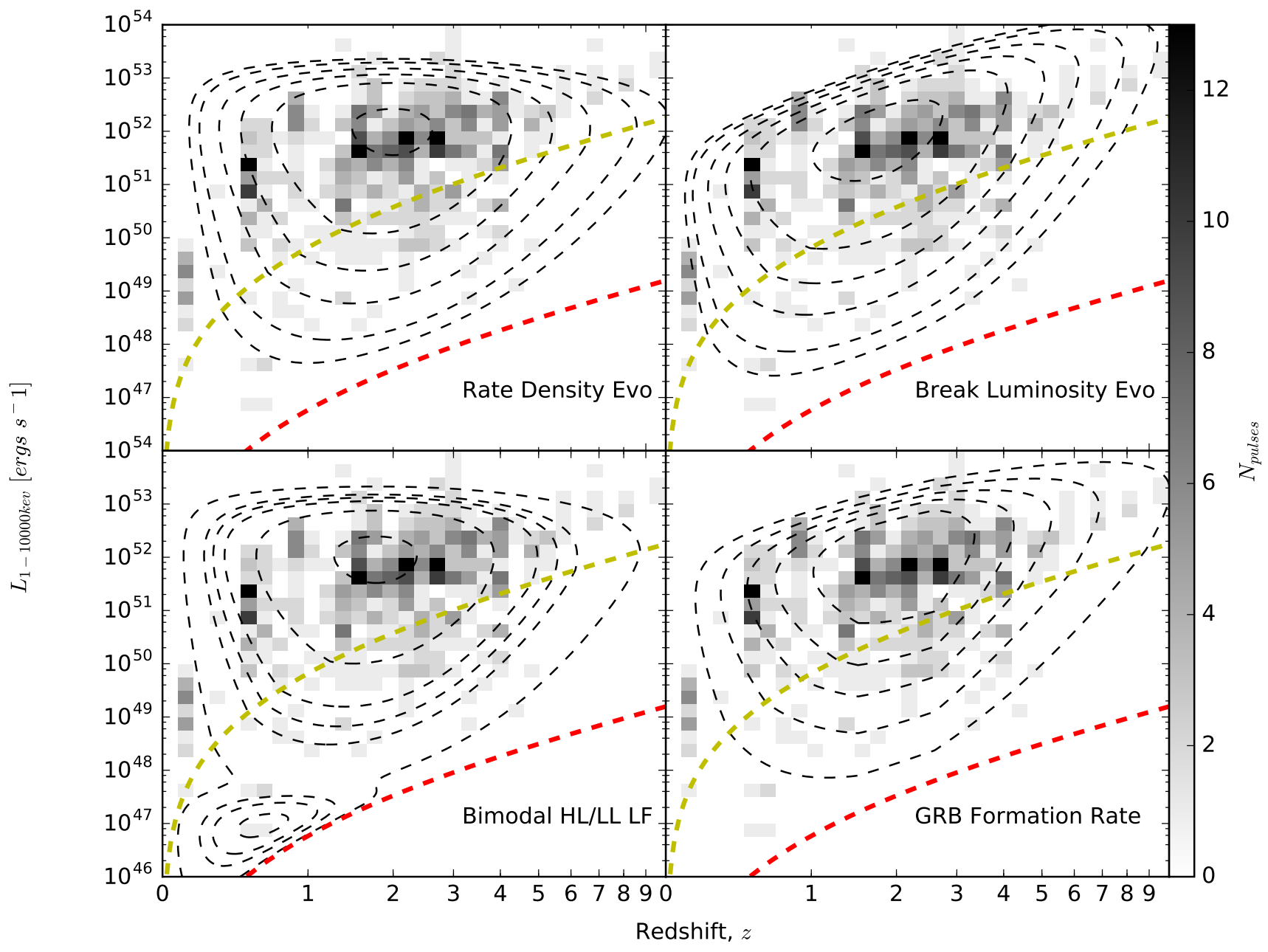

Figure 6. The derived GRB pulse luminosity distribution for a PLEC LF (dashed contours) overlaying the observed pulse distribution, binned to an arbritrary $1 / 4$ dex in luminosity and $1 / 32$ dex in redshift. The four models shown corresponds to: a type I-2 rate density evolution model, incorporating an additional $(z+1)^{\gamma}$ evolutionary factor (top left); a type I-3 break luminosity model evolving as $L_{b}=L_{0}(z+1)^{\delta}$ (top right), a type II bimodal GRB luminosity function consisting of separate high and low luminosity components (bottom left); and a type III-2 GRB formation rate model with break luminosity evolution (bottom right). The upper (yellow) and lower (red) dashed lines denote the detection thresholds of the BAT and XRT respectively.

Fryer \& Ramirez-Ruiz (2002); Firmani et al. (2004); Kocevski \& Liang (2006); Salvaterra \& Chincarini (2007); Salvaterra et al. (2009)). The BPL and PLEC LF parameters of $\alpha, \beta=\left[-0.70_{-0.06}^{+0.06},-1.69_{-0.56}^{+0.56}\right]$ and $L_{0}=[2.0 \times$ $\left.10^{50}, 1.5 \times 10^{51}\right]$ ergs $s^{-1}$ are likewise in concordance with those found in the literature, with shallower low-luminosity gradients generally derived by studies that incorporate the fainter bursts/pulses detectable by Swift.

\subsection{Evolving LF and Rate Density (Type I-5)}

We derive values of $\gamma=0.49_{-0.25}^{+0.25}$ for both LF model types and $\delta=\left[3.26_{-0.72}^{+0.72}, 2.83_{-0.55}^{+0.55}\right]$ for the BPL and PLEC, with a corresponding $\chi_{r}^{2}$ of 1.00 , and 1.04 respectively, with the majority of this improvement is seen in the very high redshift bins. The derived evolutionary parameters are similar to those of type I-2, I-3 univariate models, and suggests weak degeneracy between the rate density and break luminosity model parameters, with the evolution of the break performing the lion's share of log-likelihood optimisation. The combined Akaike weights makes the type I- 5 bivariate evolving model more than 4 times as likely as the type I-3 evolving LF break model despite the additional evolutionary parameters required. Although this suggests that a bivariate evolution model is preferred over a univariate evolution model, a model based solely on the evolution of the break luminosity cannot be ruled out entirely. 


\begin{tabular}{|c|c|c|c|c|c|c|c|c|c|}
\hline BPL & $Z / Z_{\odot}$ & $\begin{array}{c}K_{G R B} \\
{\left[10^{-8} \text { GRBs } M_{\odot}^{-1}\right]}\end{array}$ & $\gamma$ & $\begin{array}{c}L_{0} \\
{\left[10^{52} \text { ergs s}^{-1}\right]}\end{array}$ & $\delta$ & $\alpha$ & $\beta$ & $\chi_{r}^{2}$ & $w_{i}\left(A I C_{c}\right)$ \\
\hline I-1) & - & $1.18_{-0.09}^{+0.10}$ & - & $1.69_{-0.73}^{+1.29}$ & - & $-0.79_{-0.04}^{+0.04}$ & $-1.91_{-2.04}^{+2.04}$ & 1.81 & $1.4 \times 10^{-7}$ \\
\hline I-2) & - & $0.67_{-0.20}^{+0.28}$ & $0.51_{-0.30}^{+0.30}$ & $1.70_{-0.71}^{+1.22}$ & - & $-0.78_{-0.04}^{+0.04}$ & $-1.88_{-1.12}^{+1.12}$ & 1.78 & $2.6 \times 10^{-7}$ \\
\hline I-3) & - & $1.20_{-0.09}^{+0.10}$ & - & $0.02_{-0.02}^{+0.05}$ & $3.35_{-0.74}^{+0.74}$ & $-0.70_{-0.06}^{+0.06}$ & $-1.69_{-0.56}^{+0.56}$ & 1.05 & 0.0961 \\
\hline $\mathrm{I}-4)$ & $\begin{array}{c}0.01 \\
0.1 \\
0.2 \\
0.3 \\
0.4 \\
0.5 \\
0.6\end{array}$ & $\begin{array}{c}653.0_{-65.6}^{+66.7} \\
14.3_{-1.1}^{+1.2} \\
4.91_{-0.39}^{+0.43} \\
2.87_{-0.23}^{+0.25} \\
2.07_{-0.17}^{+0.18} \\
1.67_{-0.14}^{+0.15} \\
1.47_{-0.12}^{+0.13}\end{array}$ & $\begin{array}{l}- \\
- \\
- \\
- \\
- \\
- \\
-\end{array}$ & $\begin{array}{l}1.21_{-0.47}^{+0.77} \\
1.05_{-0.43}^{+0.74} \\
1.05_{-0.45}^{+0.77} \\
1.26_{-0.53}^{+0.92} \\
1.46_{-0.60}^{+1.00} \\
1.67_{-0.64}^{+1.04} \\
1.70_{-0.89}^{+1.86}\end{array}$ & $\begin{array}{l}- \\
- \\
- \\
- \\
- \\
- \\
-\end{array}$ & $\begin{array}{l}-0.75_{-0.06}^{+0.06} \\
-0.75_{-0.06}^{+0.06} \\
-0.75_{-0.06}^{+0.06} \\
-0.76_{-0.05}^{+0.05} \\
-0.77_{-0.04}^{+0.04} \\
-0.77_{-0.04}^{+0.04} \\
-0.77_{-0.04}^{+0.04}\end{array}$ & $\begin{array}{l}-1.69_{-0.23}^{+0.23} \\
-1.59_{-0.22}^{+0.22} \\
-1.55_{-0.27}^{+0.27} \\
-1.60_{-0.43}^{+0.43} \\
-1.70_{-0.48}^{+0.48} \\
-1.83_{-0.55}^{+0.55} \\
-1.89_{-4.33}^{+4.33}\end{array}$ & $\begin{array}{l}1.67 \\
1.69 \\
1.71 \\
1.71 \\
1.71 \\
1.70 \\
1.71\end{array}$ & $\begin{array}{l}5.1 \times 10^{-6} \\
4.6 \times 10^{-6} \\
4.5 \times 10^{-6} \\
4.7 \times 10^{-6} \\
5.6 \times 10^{-6} \\
6.3 \times 10^{-6} \\
5.4 \times 10^{-6}\end{array}$ \\
\hline I-5) & - & $0.69_{-0.18}^{+0.24}$ & $\begin{array}{r}0.49_{-0.25}^{+0.25} \\
\end{array}$ & $\begin{array}{r}0.02_{-0.02}^{+0.06} \\
\end{array}$ & $\begin{array}{r}3.26_{-0.72}^{+0.72} \\
\end{array}$ & $-0.70_{-0.06}^{+0.06}$ & $-1.67_{-0.72}^{+0.72}$ & 1.00 & 0.3029 \\
\hline PLEC & $Z / Z_{\odot}$ & $\begin{array}{c}K_{G R B} \\
{\left[10^{-8} \text { GRBs } M_{\odot}^{-1}\right]}\end{array}$ & $\gamma$ & $\begin{array}{c}L_{0} \\
{\left[10^{52} \text { ergs s}^{-1}\right]}\end{array}$ & $\delta$ & $\alpha$ & & $\chi_{r}^{2}$ & $w_{i}\left(A I C_{c}\right)$ \\
\hline $\mathrm{I}-1)$ & - & $1.13_{-0.10}^{+0.10}$ & - & $\begin{array}{l}4.31_{-0.92}^{+1.18} \\
\end{array}$ & - & $-0.75_{-0.05}^{+0.05}$ & - & 1.83 & $2.0 \times 10^{-7}$ \\
\hline $\mathrm{I}-2)$ & - & $0.65_{-0.18}^{+0.25}$ & $0.51_{-0.28}^{+0.28}$ & $4.55_{-1.02}^{+1.32}$ & - & $-0.75_{-0.05}^{+0.05}$ & - & 1.78 & $4.8 \times 10^{-7}$ \\
\hline I-3) & - & $1.19_{-0.10}^{+0.10}$ & - & $\begin{array}{r}0.15_{-0.07}^{+0.12} \\
\end{array}$ & $2.92_{-0.54}^{+0.54}$ & $-0.70_{-0.05}^{+0.05}$ & - & 1.08 & 0.0999 \\
\hline I-4) & $\begin{array}{c}0.01 \\
0.1 \\
0.2 \\
0.3 \\
0.4 \\
0.5 \\
0.6\end{array}$ & $\begin{array}{c}642.4_{-53.8}^{+58.7} \\
13.9_{-1.2}^{+1.3} \\
4.77_{-0.40}^{+0.44} \\
2.76_{-0.26}^{+0.26} \\
1.98_{-0.18}^{+0.18} \\
1.62_{-0.14}^{+0.15} \\
1.42_{-0.12}^{+0.13}\end{array}$ & $\begin{array}{l}- \\
- \\
- \\
- \\
- \\
- \\
-\end{array}$ & $\begin{array}{l}5.35_{-1.17}^{+1.50} \\
5.34_{-1.26}^{+1.65} \\
5.25_{-1.29}^{+1.72} \\
5.02_{-1.20}^{+1.58} \\
4.74_{-1.09}^{+1.42} \\
4.56_{-1.01}^{+1.30} \\
4.38_{-0.95}^{+1.22}\end{array}$ & $\begin{array}{l}- \\
- \\
- \\
- \\
- \\
- \\
-\end{array}$ & $\begin{array}{l}-0.76_{-0.05}^{+0.05} \\
-0.76_{-0.05}^{+0.05} \\
-0.76_{-0.05}^{+0.05} \\
-0.75_{-0.05}^{+0.05} \\
-0.75_{-0.05}^{+0.05} \\
-0.74_{-0.05}^{+0.05} \\
-0.74_{-0.05}^{+0.05}\end{array}$ & $\begin{array}{l}- \\
- \\
- \\
- \\
- \\
- \\
-\end{array}$ & $\begin{array}{l}1.79 \\
1.80 \\
1.79 \\
1.75 \\
1.73 \\
1.72 \\
1.72\end{array}$ & $\begin{array}{l}6.2 \times 10^{-7} \\
5.2 \times 10^{-7} \\
9.0 \times 10^{-7} \\
2.1 \times 10^{-6} \\
3.8 \times 10^{-6} \\
4.1 \times 10^{-6} \\
3.7 \times 10^{-6}\end{array}$ \\
\hline I-5) & - & $0.68_{-0.07}^{+0.23}$ & $0.49_{-0.24}^{+0.24}$ & $0.17_{-0.08}^{+0.14}$ & $2.83_{-0.55}^{+0.55}$ & $-0.70_{-0.05}^{+0.05}$ & - & 1.04 & 0.5010 \\
\hline
\end{tabular}

Table 1. Fitted results for Type I models, where the GRB pulse formation rate function, $\Psi_{*}(z)$, incorporates a Cole CSFRD parameterised by Kobayashi, Inoue \& Inoue (2013). The best fit parameters for the BPL and PLEC LF models are shown for the scenarios of I-1) where there is no extra evolutionary parameter $(\iota(z)=1)$; I-2) there is evolution in the GRB formation rate $\left(\iota(z) \propto(z+1)^{\gamma}\right)$; I-3) there is evolution in the break luminosity $\left(L_{\text {break }} \propto(z+1)^{\delta}\right)$; I-4) there is present a metallicity density evolution $(\iota(z)=\Sigma(z)$ and $\Sigma(z)=$ $\left.\hat{\Gamma}\left[0.84,\left(Z / Z_{\odot}\right)^{2} 10^{0.3 z}\right] / \Gamma(0.84)\right)$ and; I-5) there is simultaneously evolution of the rate density and break luminosity $\left(\iota(z) \propto(z+1)^{\gamma}\right.$ and $\left.L_{\text {break }} \propto(z+1)^{\delta}\right)$. The $\chi_{r}^{2}$ values, and Akaike weights, $w_{i}\left(A I C_{c}\right)$, are derived from the bins shown in Figure 4 , and are quoted as a means of comparison rather than the means of fitting. A perfect fit is almost impossible due to the clumpy nature of the pulse data coupled with the affect that uncertainties in the larger bins have on the likelihood.

\subsection{Redshift, Luminosity, and Flux Cuts}

In all single population GRB pulse models the residuals of fitted GRB luminosity functions are greatest at the extrema of the GRB pulse $L-z$ distribution:

- non-evolving models underestimate the population of high$z$, high- $L$ pulses, whilst overestimating that of low- $z$, low- $L$ pulses;

- rate density models overestimate the population of high- $z$, high- $L$ pulses, whilst underestimating that of low- $z$, low- $L$ pulses;

- both models that incorporate break luminosity overestimate the high- $z$, high- $L$ GRB pulse populations but are consistent with their large associated uncertainties, contributing little to the log-likelihood function.

Discrepencies at the extrema may be due to parent GRBs that are significantly different from the bulk population, ei- ther through a separate GRB progenitor type (Pop III stars for high- $z$, high- $L$ GRBs) or a via a more complex GRB luminosity function (LL \& HL GRBs). Cutting away GRB pulses that lie in the extrema of the redshift, or luminosity distributions may produce noticible changes in fitted parameters, suggestive of LGRB sub-populations. We find, however, that performing successive cuts in the data for the type I-5 PLEC LF model (see Figure 7) of the high/low regions (filled/empty circles) of the $z$, or $L$ distributions (black/green data) produces weak variations in the fitted parameters, which becomes more pronounced as the sample size decreases. Such variations in the fitted parameters are, however, small with good overlap of the $1 \sigma$ confidence intervals. Whilst this suggests that the low $z /$ high- $z$, and low- $L /$ high- $L$ GRB pulses are part of a single population rather than belonging to unique sub-populations, we cannot rule out the possibility that the latter is true. 


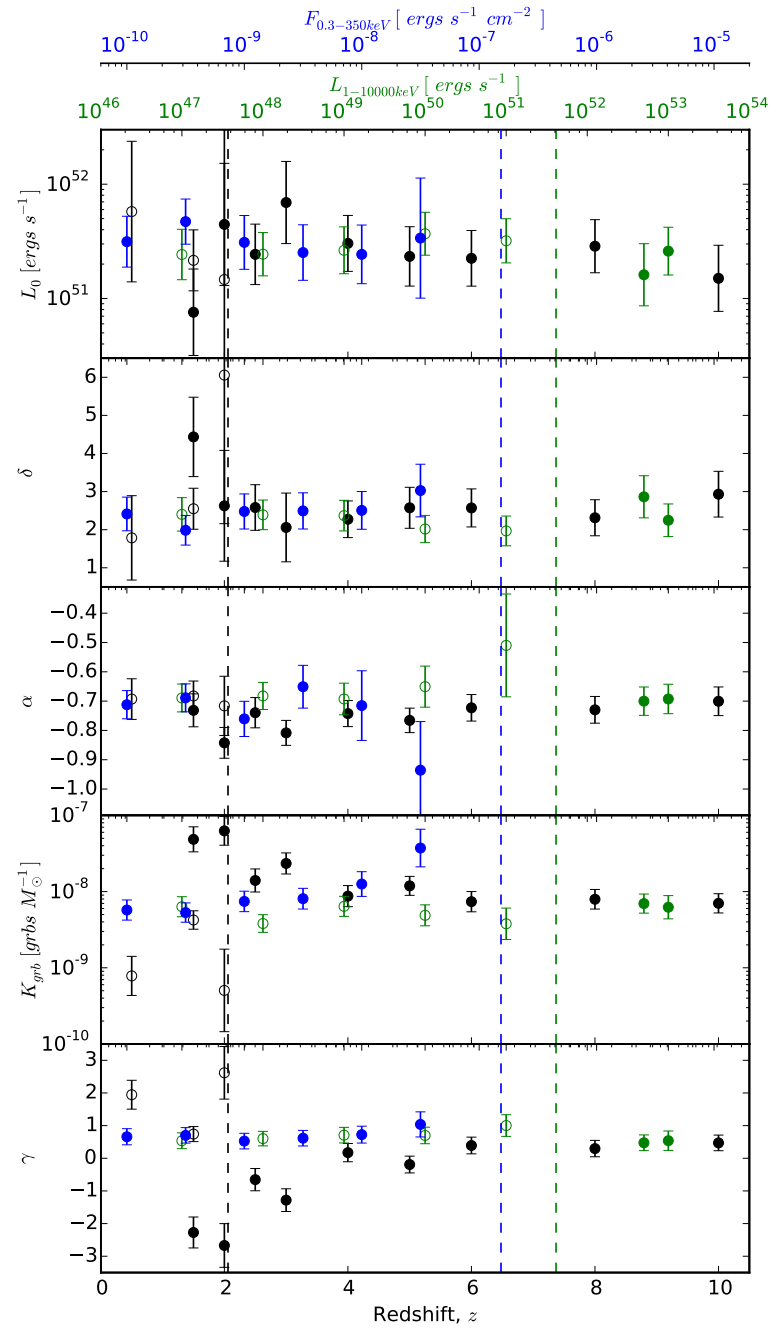

Figure 7. Fitted MCMC parameters of a fully evolving type I-5 PLEC LF model with successive redshift cuts (black axis), bolometric rest-frame luminosity cuts (green axis), or observer-frame integrated peak flux cuts (blue axis) to the GRB pulse data. Filled and empty circles denote the upper and lower cutoffs respectively for that particular dataset with $1 \sigma$ uncertainties. Overlaid are the median redshift, luminosities, and fluxes of the pulse data (dashed lines).

It is common in the data selection phase of GRB luminosity studies to apply a flux cut to the data, with authors arguing that the brightest GRBs in the observer-frame suffer the least from detection bias, and as such are more representative of the true population of GRBs. The study by Salvaterra et al. 2012, for example, utilises a flux cut of $P_{15-150 \mathrm{keV}}=2.6$ photons $\mathrm{s}^{-1} \mathrm{~cm}^{-2}$ in the observer frame, equating to an integrated flux of $F_{15-150 \mathrm{keV}} \approx 1.21 \times 10^{-5}$ ergs $\mathrm{s}^{-1} \mathrm{~cm}^{-2}$ for a PLEC spectrum with $\alpha=-1.57$, $E_{c}=183 \mathrm{keV}$. The inclusion of a high flux limit has led to suggestions that the observed evolution seen in such studies arise from a flux threshold selection effect rather than being an intrinsic property of the GRB luminosity function (Howell \& Coward 2013). To this end, we vary the flux selection threshold on our GRB pulse data and re-run our MCMCs to refit the data. We find some variation in the GRB pulse luminosity fit parameters (Figure 7 , blue data) however such variation, like those of the redshift and luminosity thresholds, are consistent with the intrinsic uncertainties of the model fit parameters. Whilst the direction of the rate evolution parameter, $\gamma$, varies in direction such that it is not concrete that such evolution is real, the evolution in the break luminosity is strong and sees little variation when applying various data cuts.

\section{THE TYPE II GRB MODEL}

To reduce the dimensionality of the bimodal LL \& HL GRB LF model, we fix the indexes of the two populations at the values derived for a Type I-1, non-evolving single population GRB LF, such that $\alpha=\alpha_{H L}=\alpha_{L L}\left(\right.$ or $\left.\beta=\beta_{H L}=\beta_{L L}\right)$. We find little variation between the HL LF parameters and the single population Type I-1 LF parameters, unsurprising given the bulk of the GRB pulse population lies within the regime of HL GRBs. We find a local HL GRB formation rate density, $\rho_{0}^{H L}$ of $0.22_{-0.02}^{+0.02}, 0.21_{-0.02}^{+0.01}$ GRBs $G p c^{-3}$ $y r^{-1}$ for the PLEC and BPL LFs respectively, compared to the $0.09<\rho_{0}^{H L}<1.2 \mathrm{GRBs} G p c^{-3} y r^{-1}$ range found by Liang et al. (2007); Virgili, Liang \& Zhang (2009); Howell \& Coward (2013) for their high luminosity GRBs. The inclusion of a secondary LL LF marginally improves the fitting of the observed $L-z$ GRB pulse distribution (see bottom left panel, Figure 6), reducing the $\chi_{r}^{2}$ contribution of the low- $L$, low- $z$ bins at the expense of twice the number of input parameters. The secondary LL GRB pulse LF shows a break at $L_{0}^{L L}=7.21 \times 10^{46}, 1.10 \times 10^{47} \mathrm{ergs} s^{-1}$ for the BPL and PLEC LF models respectively, with a local GRB formation rate density of $\rho_{0}^{L L}=0.09,0.21 \mathrm{GRBs} G p c^{-3} \mathrm{yr}^{-1}$.

The ratio of low/high-luminosity GRB formation rate densities found in this paper are approximately at unity, compared to the ratios of $50-200$ found in favour of LL GRBs (Liang et al. 2007; Virgili, Liang \& Zhang 2009; Howell \& Coward 2013); varying the limits of normalisation of the LFs has a small effect on the derived $K_{G R B}$ efficiencies and is not a solution to the discrepency. Despite a sample of 72 GRB pulses, we are unable to constrain uncertainties in the fitted parameters. Although reproducing the observed $L-z$ GRB pulse distribution, the overall combined Akaike weights for the Type II models $\left(w_{i}\left(A I C_{C}\right)=0.1332\right)$, versus the non-evolving Type I-1 LF models $\left(w_{i}\left(A I C_{C}\right)=0.8668\right)$ strongly suggests that a single, non-evolving population of GRB pulses is a better representation of the $L-z$ distribution and is not a suitable explanation to the observed evolution of the break luminosity. We do not rule out that LL GRB pulses are a separate subgroup, however our data does suggest that it is highly unlikely.

\section{THE TYPE III GRB MODELS}

\subsection{No Evolution Model (Type III-1)}

We find that our fit utilising the Type III-1 GRB formation rate model, with $\chi_{r}^{2}$ values of 2.14 , and 2.13 , produces a strong rise in GRB pulse formation rates from the current epoch, plateauing at $z=1.5$, before decaying strongly away at $z=2.6$. This follows a similar shape as the CSFRD and produces similar $L_{0}, \alpha$, and $\beta$ values as the equivalent Type I/II models fitted in Section 5, and 6. On initial inspection 


\begin{tabular}{lcc}
\hline BPL & Type I-1 & Type II \\
\hline$K_{G L B}^{H L}$ & $1.18_{-0.09}^{+0.10} \times 10^{-8}$ & $1.18_{-0.09}^{+0.09} \times 10^{-8}$ \\
$L_{0}^{H L}$ & $1.69_{-0.73}^{+1.29} \times 10^{52}$ & $1.70_{-0.32}^{+0.40} \times 10^{52}$ \\
$\alpha^{H L}$ & $-0.79_{-0.04}^{+0.04}$ & -0.79 \\
$\beta^{H L}$ & $-1.94_{-2.04}^{+2.04}$ & -1.94 \\
$K_{G R B}^{L L}$ & - & $0.50 \times 10^{-8}$ \\
$L_{0}^{L L}$ & - & $7.21 \times 10^{46}$ \\
$\alpha^{L L}$ & - & -0.79 \\
$\beta^{L L}$ & - & -1.94 \\
$\chi_{r}^{2}$ & 1.81 & 1.91 \\
$w_{i}\left(A I C_{C}\right)$ & 0.3587 & 0.0088 \\
\hline $\mathrm{PLEC}$ & $\mathrm{Type} \mathrm{I}-1$ & $\mathrm{Type} \mathrm{II}$ \\
\hline$K_{G L}^{H L}$ & $1.13_{-0.10}^{+0.10} \times 10^{-8}$ & $1.12_{-0.09}^{+0.10} \times 10^{-8}$ \\
$L_{0}^{H L}$ & $4.31_{-0.92}^{+1.18} \times 10^{52}$ & $4.23_{-0.71}^{+0.85} \times 10^{52}$ \\
$\alpha^{H L}$ & $-0.75_{-0.05}^{+0.05}$ & -0.75 \\
$K_{G}^{L L}$ & - & $1.11 \times 10^{-8}$ \\
$L_{0}^{L L}$ & - & $1.10 \times 10^{47}$ \\
$\alpha^{L L}$ & - & -0.75 \\
$\chi_{r}^{2}$ & 1.83 & 1.85 \\
$w_{i}\left(A I C_{C}\right)$ & 0.5081 & 0.1244 \\
\hline
\end{tabular}

Table 2. The fitted Type I-1, non-evolving model GRB LF for a single population of GRBs (Table 1) and a Type II, bimodal population of high-luminosity $\left(L>10^{50} \mathrm{ergs}^{-1}\right)$ and low-luminosity $\left(L<10^{50}\right.$ ergs $\left.s^{-1}\right)$ GRBs. $K_{G R B}$ is given in units of GRBs $M_{\odot}^{-1}$ and $L_{0}$ is in units of ergs $s^{-1}$. Uncertainties in $\alpha$ and $\beta$ for the bimodal population are not given as these parameters were fixed beforehand. The fitted LL GRB LF parameters are quoted without associated errors as they are unconstrained. The $\chi_{r}^{2}$ values, and Akaike weights, $w_{i}\left(A I C_{c}\right)$, are derived from the bins shown in Figure 4.

the Type III-1 models perform less well in fitting, as it requires more than twice the number of model parameters to achieve similar likelihoods, and suffers from the same inability to reproduce GRB pulse formation rates at the extrema of the $L-z$ pulse distribution as that of extra rate evolution for Type I-2 models. Whilst this would lead to one assuming that a phemonenological model is better than an empirical one, it is important to note that the CSFRD models have significant uncertainties which are almost universally ignored when propagating errors, creating a false impression of greater quality; it is for this reason that we do not cast any favourable opinion on Type I over Type III models. The combined Akaike weight of $A I C_{c}=5.61 \times 10^{-6}$ for both nonevolving type III-1 LF models reinforces the conclusion that evolution in the break luminosity are required to reproduce the observed pulse $L-z$ distribution. This becomes more clear when looking at the probability, and cumulative density functions (Figure 8); the dashed lines, corresponding to a non-evolving type III-1 model produces a CDF that fails to reproduce the clear luminosity evolution seen across the three redshift bins, with distinct underestimation of the luminosities of high- $z$ pulses, and overestimation of the luminosities of low- $z$ pulses.

Our pulse luminosity function is consistent with other studies that fit a triple power-law to the GRB formation rate. Although we utilise all pulses within a GRB lightcurve and our redshift breaks in the GRB formation rate differ, we find good agreement with the low-redshift, and high-redshift indexes of Butler, Bloom \& Poznanski (2010)

\begin{tabular}{lcc}
\hline BPL & Type III-1 & Type III-2 \\
\hline$a_{1}$ & $2.69_{-0.45}^{+0.45}$ & $2.48_{-0.90}^{+0.90}$ \\
$z_{1}$ & 1.5 & 1.5 \\
$a_{2}$ & $0.08_{-0.33}^{+0.33}$ & $0.23_{-0.51}^{+0.51}$ \\
$z_{2}$ & 2.6 & 2.6 \\
$a_{3}$ & $-1.83_{-1.01}^{+1.01}$ & $-1.97_{-1.43}^{+1.43}$ \\
$\rho_{0}$ & $7.02_{-2.52}^{+3.94} \times 10^{-2}$ & $8.29_{-4.10}^{+8.13} \times 10^{-2}$ \\
$L_{0}$ & $1.71_{-0.39}^{+0.52} \times 10^{52}$ & $0.15_{-0.09}^{+0.20} \times 10^{52}$ \\
$\delta$ & - & $2.04_{-0.45}^{+0.45}$ \\
$\alpha$ & $-0.72_{-0.12}^{+0.12}$ & $-0.69_{-0.09}^{+0.09}$ \\
$\beta$ & $-1.79_{-0.22}^{+0.22}$ & $-1.88_{-0.25}^{+0.25}$ \\
$\chi_{r}^{2}$ & 2.14 & 1.43 \\
$w_{i}\left(A I C_{C}\right)$ & $1.1 \times 10^{-7}$ & 0.0415 \\
\hline PLEC & Type III-1 & Type III-2 \\
\hline$a_{1}$ & $2.23_{-1.32}^{+1.32}$ & $2.39_{-1.25}^{+1.25}$ \\
$z_{1}$ & 1.5 & 1.5 \\
$a_{2}$ & $0.73_{-0.38}^{+0.38}$ & $0.85_{-0.44}^{+0.44}$ \\
$z_{2}$ & 2.6 & 2.6 \\
$a_{3}$ & $-2.20_{-0.95}^{+0.95}$ & $-2.27_{-0.82}^{+0.82}$ \\
$\rho_{0}$ & $7.98_{-5.23}^{+15.23} \times 10^{-2}$ & $7.42_{-4.86}^{+14.06} \times 10^{-2}$ \\
$L_{0}$ & $4.02_{-0.96}^{+1.25} \times 10^{52}$ & $0.36_{-0.21}^{+0.48} \times 10^{52}$ \\
$\delta$ & - & $2.06_{-0.70}^{+0.70}$ \\
$\alpha$ & $-0.71_{-0.07}^{+0.07}$ & $-0.66_{-0.06}^{+0.06}$ \\
$\chi_{r}^{2}$ & 2.13 & 1.42 \\
$w_{i}\left(A I C_{C}\right)$ & $5.5 \times 10^{-6}$ & 0.9585 \\
\hline & &
\end{tabular}

Table 3. The fitted parameters for the Type III GRB LF models using a triple broken power-law function that is directly fitted to the GRB formation rate, either excluding (Type III-1) or including (Type III-2) evolution of the LF break. $a_{1}, a_{2}$, and $a 3$ are the gradients of the three power-laws; $z_{1}$, and $z_{2}$ are the redshift breaks, set at the bin edges discussed in Section 4.1 ; and $\rho_{0}$ is the GRB formation rate density at redshift $z=0 . L_{0}$ is given in units of ergs $s^{-1}$, and $\rho_{0}$ in units of GRBs $G p c^{-3} y r^{-1}$.

(BBP), and Wanderman \& Piran (2010) (WP). We derive an $\alpha_{1}$, and $\alpha_{3}$ of $2.69_{-0.45}^{+0.45},-1.83_{-1.01}^{+1.01}$ respectively, compared to: $3.35_{-0.74}^{+0.74},-2.51_{-2.25}^{+1.60}(\mathrm{BBP})$; and $3.1_{-0.7}^{+0.7},-2.9_{-2.4}^{+1.6}$ (WP) for their models that include GRBs with known redshifts. The intermediate-redshift indexes, $\alpha_{2}$, derived by those studies $\left(1.32_{-0.58}^{+0.58}, \mathrm{BBP}\right.$; and $\left.1.4_{-0.6}^{+0.6}, \mathrm{WP}\right)$ are significantly stronger than the $0.08_{-0.33}^{+0.33}$ we find, and can be explained by the difference in position of the first redshift break those authors utilise, who, like ourselves, do not set as a free parameter in their fitting.

Our luminosity functions produce a stronger lowluminosity index than these studies, possibly due to the large number of low-luminosity BAT and XRT pulses we incorporate, with $\alpha=-0.72_{-0.12}^{+0.12}$ compared to $-0.27_{-0.19}^{+0.19}(\mathrm{BBP})$, and $0.22_{-0.31}^{+0.18}(\mathrm{WP})$. Our low-luminosity index is however consistent both with our Type I and Type II models, and other studies that utilise a CSFRD. The high-luminosity index, $\beta$, derived by Butler, Bloom \& Poznanski (2010) at $-3.46_{-1.53}^{+1.53}$ is significantly stronger than our own derived results of $-1.79_{-0.22}^{+0.22}$; however our results are consistent with $-1.4_{-0.6}^{+0.3}$ of Wanderman \& Piran (2010) and is most likely due to both our studies utilising peak luminosities rather than the time-averaged luminosities used by Butler, Bloom \& Poznanski (2010). The break luminosity, $L_{0}$, derived in this paper at $10^{52.23_{-0.12}^{+0.12}}$ ergs $s^{-1}$ is lower than either stud- 


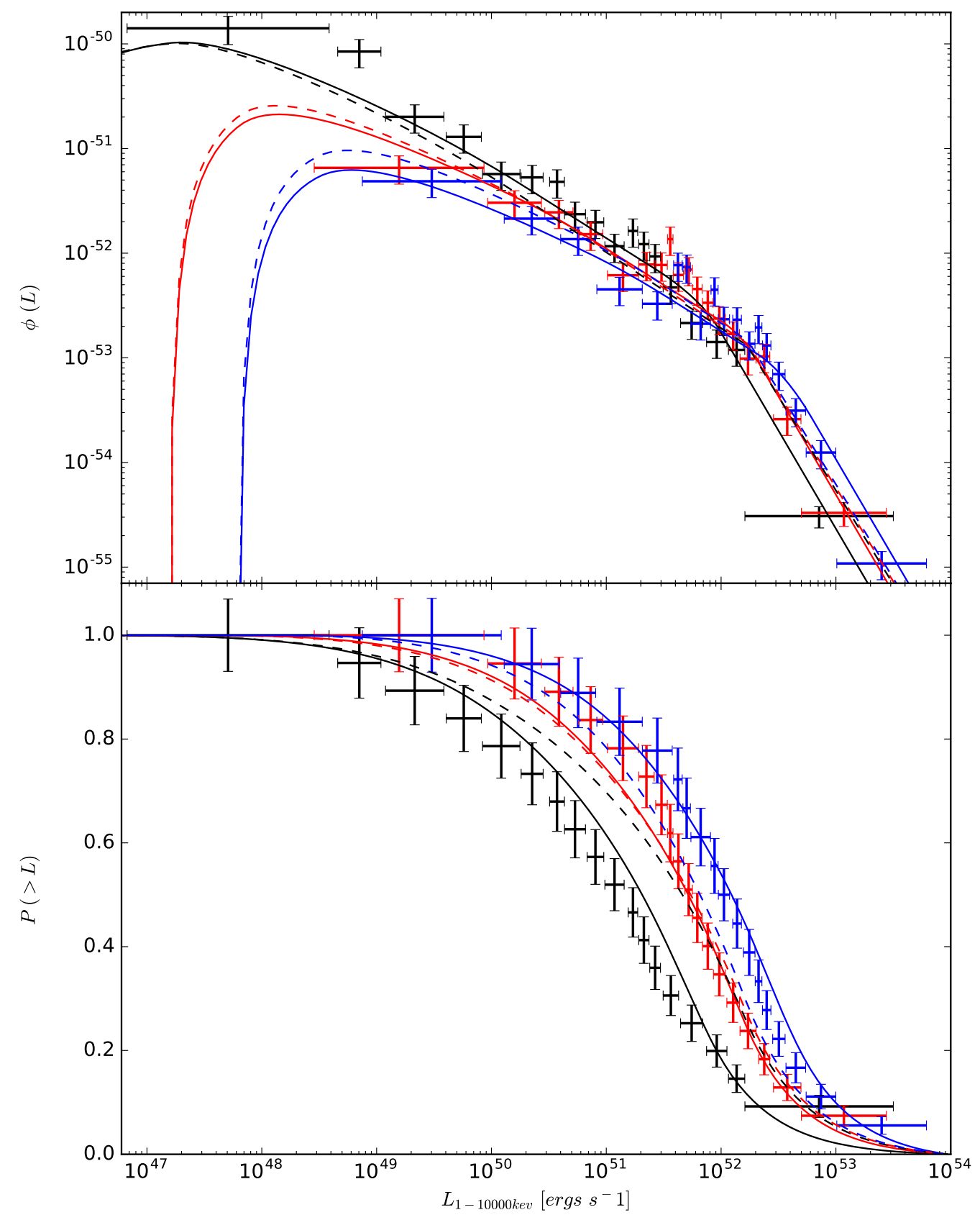

Figure 8. The observed, and derived GRB pulse probability densities (top) for the three redshift bins, and the equivalent cumulative probability densities (bottom) for a type III GRB pulse formation models with a broken power-law LPDF. Black data, and lines correspond to the $0.125<z \leqslant 1.505$ whilst red, and blue data correspond to the $1.51<z \leqslant 2.6$ and $2.612<z \leqslant 9.4$ bins respectively. The solid lines denote the type III-2 model with an additional $(z+1)^{\delta}$ evolution in the break of the LPDF, whilst the dashed line is a non-evolving type III-1 model. The turn-off at low luminosities is due to the convolution to the Swift detection profile. 
ies finds and is consistent with $10^{52.5_{-0.2}^{+0.2}}$ ergs $s^{-1}$ (WP) but not with $10^{52.95_{-0.31}^{+0.31}}(\mathrm{BBP})$.

\subsection{Break Luminosity Evolution (Type III-2)}

Like the Type I-3 model, inclusion of evolution in the break of the luminosity function significantly improves the quality of fits $\left(\chi_{r}^{2}=1.43,1.42\right.$ for the BPL, and PLEC respectively), with improvement at the high- $L$, high- $z$ extrema of the pulse $L-z$ distribution (bottom right panel, Figure 6 ). With a combined Akaike weight of $w_{i}\left(A I C_{C}\right) \sim 1$ for the BPL, and PLEC models, the evolving Type III-2 luminosity functions are $1.7 \times 10^{5}$ times more likely than the non-evolving Type III-1 models to reproduce the observed pulse distribution. As shown in Figure 8, the CDF for a BPL LPDF model with break luminosity evolution is able to reproduce the observed CDFs for all redshift bins, within $1 \sigma$ uncertainties. The evolutionary index of the break luminosity, at $\delta=2.04_{-0.45}^{+0.45}$, and $2.06_{-0.7}^{+0.7}$ for the BPL, and PLEC models is weaker than that found using the fully evolving Type I-5 model but remains consistent with other Swift studies (see Section 5.4 for references).

\section{LGRBS AND THE CSFRD}

The fitted GRB formation rate densities for Type I-5 and Type III-1 models derived in this paper are shown in Figure 9 overlain with observed cosmic star formation rate densities, the parameterised CSFRD model of Hopkins \& Beacom (2006), and GRB formation rate models of Salvaterra et al. (2012); Butler, Bloom \& Poznanski (2010); Wanderman \& Piran (2010). Normalised to the CSFRD, our Type I-5 (including rate and luminosity evolution) and Type III-1 models trace the observed CSFRD well, especially at low/intermediate redshifts $(z<5)$, with up to a factor 2 deviation between derived high- $z(z>5)$ pulse rates and cosmic star formation rates for the Type III-1 model; suggestive of a redshift break at an earlier epoch than that which was assumed.

The parameterised model of Hopkins \& Beacom (2006), common in the GRB literature as a model for CSFRD, traces the CSRFD at low redshifts, with noticible drop-off at highredshifts. Assuming that the GRB formation rate follows the CSFRD only, requires the addition of GRB formation rate evolution to the Type I-1 models to boost high- $z$ GRB formation rates. This addition may, however, suggest that the parameterised CSFRD models are incorrect at high- $z$ rather than implying the rate of GRB formation was greater at earlier epochs. Comparing the performance of our rate density evolving Type I-2 model, and the CSFRD as parameterised by Hopkins \& Beacom (2006) to the observed CSFRD shows that our Type I-2 model with rate density produces a loglikelihood of 106.67 compared to a log-likelihood of -17.78 for Hopkins \& Beacom (2006). The corresponding Akaike weights for the Type I-2 model is $\sim 1.0^{9}$, indicating that the

9 The log-likelihood for the Type I-2 model is calculated after the unknown conversion factor from GRB formation rate density to CSFRD is accounted for, and as such the comparison is with regards to the shape of the CSFRD.
GRB formation rate evolution seen is not real but is, instead, an artifact of utilising inappropriate CSFRD models. Utilising GRB formation rates as a probe to high- $z$ star-formation is therefore highly speculative: conversion from GRB formation rates to star-formation rates are often cyclical; a starformation rate and GRB evolution rate are assumed in order to derive a GRB formation rate, with which a star-formation rate is derived (Kistler et al. 2008; Robertson \& Ellis 2012); as such, careful consideration is required when attempting to derive CSFRD models using high- $z$ burst rates.

\subsection{The Local GRB Formation rate}

The GRB formation efficiency parameter, in combination with the star formation rate density at $z=0$, produces the local GRB formation rate density, $\rho_{0}$. For the Type I models excluding rate density or metallicity density evolution (I-1, I-3), the formation efficiency, $K_{G R B}$, was derived to be $K_{G R B}=1.18_{-0.10}^{+0.10} \times 10^{-8} \mathrm{GRBs} M_{\odot}^{-1}$, in good agreement to the values of $1.07 \pm 0.11 \times 10^{-8}$ and $1.05 \pm 0.05 \times 10^{-8}$ GRBs $M_{\odot}^{-1}$ (Salvaterra \& Chincarini 2007; Salvaterra et al. 2009). This equates to a local formation rate density of $\rho_{0}=0.22_{-0.02}^{+0.02} \mathrm{GRBs} G p c^{-3} y r^{-1}$; for models including rate density (I-2, I-4) this drops to $\rho_{0}=0.12_{-0.04}^{+0.05}$ GRBs $G p c^{-3}$ $y r^{-1}$. For the Type III models, the local GRB formation rate is one of the model parameters, and for a non-evolving BPL LF model this produces a $\rho_{0}$ of $0.07_{-0.03}^{+0.04} \mathrm{GRBs} G p c^{-3} y r^{-1}$, increasing to $0.083_{-0.04}^{+0.08}$ GRBs $G p c^{-3} y r^{-1}$ for a Type III-2 evolving BPL LF model. These values are towards the lower end of the distribution of values found in the literature for models excluding jet-beaming $\left(0.03<\rho_{0}<7.3\right.$ GRBs $G p c^{-3}$ $y r^{-1}$ Porciani \& Madau (2001); Schmidt (2001); Guetta, Piran \& Waxman (2005); Wanderman \& Piran (2010); Cao et al. (2011); Salvaterra et al. (2012); Yu et al. (2015)).

\section{CONCLUSIONS}

The lightcurves of Gamma-ray bursts exhibit wide variation in temporal fluctuations with some showing single, bright FRED-like profiles whilst others have multiple peaks, often with significant overlap. Utilising a physically motivated model (Willingale et al. 2010) that considers the entire prompt, and late-time emission as a series of pulses and/or flares with corresponding emission spectra modelled by a power-law with exponential cutoff, and in most cases an afterglow component, we fit 118 LGRBs covering the period from 26/01/05 - 03/05/11. This produced 607 GRB pulses spanning $10^{46}<L<10^{54}$ ergs $s^{-1}$ in bolometric luminosity, with known redshifts up to $z \sim 10$.

Traditionally, the brightest pulse of a GRB with known redshift is used as the defining luminosity of the burst. Such pulses however do not exhibit any other unique quality: they are often not the first pulse to trigger the BAT, nor do they solely occur within the prompt emission; they do not possess the hardest spectrum within a lightcurve, nor do they see the greatest hard-to-soft evolution of said spectrum; even their brightness is, in some cases, hardly unique as some bursts contain multiple pulses of comparable brightness. A great deal of information is therefore lost when utilising solely the brightest pulses, compounding the difficulties in population analysis for a relatively rare phenomena which, un- 


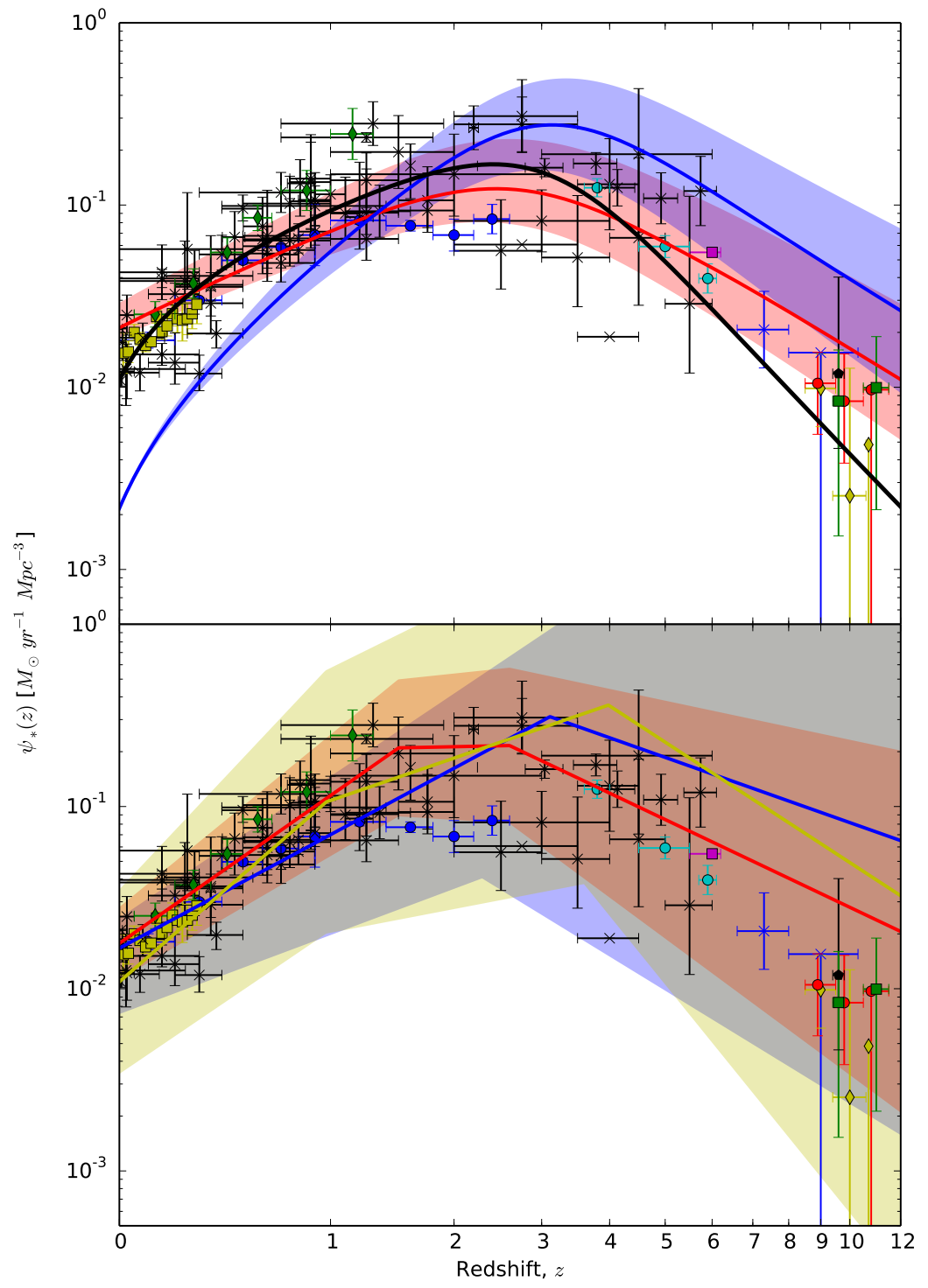

Figure 9. Observed cosmic star formation rate densities normalised to the results collated by Hopkins \& Beacom (2006) (solid black line best fit) with data from Bouwens et al. (2003) (magenta squares), Hopkins \& Beacom (2006) (black crosses), Baldry et al. (2005) (yellow squares), Pérez-González et al. (2005) (blue circles), Bouwens et al. (2007) (cyan circles), Bouwens et al. (2008) (blue crosses), Rujopakarn et al. (2010) (green diamonds), Zheng et al. (2012) (black pentagons), Coe et al. (2013) (green squares), Oesch et al. (2013) (yellow diamonds), and Ellis et al. (2013) (red circles). Overplotted for comparison are the derived GRB formation rate densities using the rate evolution GRB LF models (top panel) of Salvaterra et al. (2012) (equivalent to a Type I-2 model; PLEC LF, blue line), and the rate \& break luminosity evolution model derived in this work (Type I-5 model; PLEC LF, red line); and the direct GRB formation rate fitted LF models (bottom panel), equivalent to a Type III-1 model, of Butler, Bloom \& Poznanski (2010) (yellow line), Wanderman \& Piran (2010) (blue line), and the non-evolving Type III-1 model of this work (BPL LF, red line). Shaded regions denote $1 \sigma$ uncertainties. All GRB formation rates are are normalised to the observed star formation rates by maximising a minus log-likelihood function, producing normalisation constants, in units of $M_{\odot} G R B^{-1}$, of $6.56 \times 10^{9}$ (Salvaterra et al. 2012), and $0.17 \times 10^{9}$ (this work, Type I-5 PLEC LF model with rate \& break luminosity evolution); and $2.74 \times 10^{9}$ (Butler, Bloom \& Poznanski 2010), $1.34 \times 10^{7}$ (Wanderman \& Piran 2010), and $2.53 \times 10^{8}$ (this work, Type III-1 BPL LF model). 


\section{The Pulse Luminosity Function of Swift Gamma-ray Bursts}

til recently, was sparcely populated. We therefore choose to compute the GRB pulse luminosity function, of which the traditional GRB luminosity function can be considered as either a high-luminosity, or high-flux sub-population. We convolve a GRB pulse luminosity probability density function to a GRB formation rate model using three of the most popular GRB LF theories in the literature: Type I models that traces the cosmic star formation rate, convolved with various evolutionary effects such as break luminosity (I-3), rate density (I-2) and metallicity density evolution (I-4); Type II models that are bimodal in nature, allowing for distinct populations of low-luminosity and high-luminosity GRB pulses; and Type III models that are fitted directly to the GRB formation rate. We consider both PLEC, and BPL luminosity probability density functions, popular within the GRB literature, as they consistently produce similar quality of fits and as such neither model is preferred in our conclusions.

We find that the inclusion of rate, and metallicity density evolution, which are popular solutions to the issue of underprediction in the GRB formation rate of high- $z$ bursts, produces marginal improvement in our models however, when compared to other solutions, are entirely inadequate in explaining the observed evolution of GRB pulse luminosities. The derived GRB formation rate, either incorporating rate density evolution as a Type I-2 model, or as a Type III-1 model, traces the CSFRD up to high- $z$ and suggests that the parameterisation of CSFRD models is poor at high redshift, rather than indicating an intrinsic evolution in the GRB formation rate on top of the CSFRD. We find that within Type I or Type III models, evolution in the break of the LPDF, as a function of $(z+1)^{\delta}$, is essential to reproduce the observed $L-z$ GRB pulse distribution, with $\delta$ exhibiting a strong $(>2)$, positive evolution, consistent with studies that utilise the single brightest GRB pulses. We evaluated the possibility that this evolution in the break luminosity was down to the presence of a bimodal population of low/high luminosity GRB pulses, however our results suggest that a single population of GRBs extending from the closest, least luminous to the brightest, and furthest GRBs is preferred. We observe that Type III models consistently produce poorer fits to the data than their Type I counterparts, however we conclude that this is an artifact of assuming that components of the Type I progenitor models are known with absolute precision, which is not the case for the CSFRD. To this end we do not attempt to conclude as to the effacy of one method over another.

We conclude that treating each GRB pulse as an independent event and utilising the entire GRB pulse population in GRB LF models produces parameters in excellent agreement to those derived using the single brightest pulse within a GRB's lightcurve; it is clear that there is no advantage to using solely the brightest GRB pulse as using all GRB pulses can dramatically improve the statistics of GRB luminosity functions, and may be extended to investigating the properties of other intrinsic GRB relationships. Whilst in reality each pulse cannot be truly independent from another as they are powered from a single central engine, the relationship between bright, and faint; late, and early pulses is non-trivial.

\section{ACKNOWLEDGMENTS}

We gratefully acknowledge funding for Swift at the University of Leicester by the UK Space Agency. A.A-R's studentship funding is provided by the STFC. We thank the referee for their useful comments and suggestions.

\section{REFERENCES}

Akaike H., 1974, IEEE Transactions on Automatic Control, 19, 716

Amati L. et al., 2002, A\&A, 390, 81

Anderson T. W., Darling D. A., 1952, Ann. Math. Statist., 23, 193

Atteia J.-L., 2003, A\&A, 407, L1

Baldry I. K. et al., 2005, MNRAS, 358, 441

Barthelmy S. D. et al., 2005, Space Sci. Rev., 120, 143

Bouwens R. J., Illingworth G. D., Franx M., Ford H., 2007, ApJ, 670, 928

Bouwens R. J., Illingworth G. D., Franx M., Ford H., 2008, ApJ, 686, 230

Bouwens R. J. et al., 2003, ApJ, 595, 589

Burrows D. N. et al., 2005, Space Sci. Rev., 120, 165

Butler N. R., Bloom J. S., Poznanski D., 2010, ApJ, 711, 495

Campisi M. A., Li L.-X., Jakobsson P., 2010, MNRAS, 407, 1972

Cao X.-F., Yu Y.-W., Cheng K. S., Zheng X.-P., 2011, MNRAS, 416, 2174

Chincarini G., Moretti A., Romano P., Falcone A. D., Mor-

ris D., Racusin J., et al., 2007, ApJ, 671, 1903

Coe D. et al., 2013, ApJ, 762, 32

Cole S. et al., 2001, Monthly Notices of the Royal Astronomical Society, 326, 255

D'Agostini G., 2005, ArXiv Physics e-prints

Daigne F., Rossi E. M., Mochkovitch R., 2006, MNRAS, 372,1034

Darling D. A., 1957, Ann. Math. Statist., 28, 823

D'Avanzo P. et al., 2014, MNRAS, 442, 2342

Davé R., 2008, MNRAS, 385, 147

Deng C.-M., Wang X.-G., Guo B.-B., Lu R.-J., Wang Y.-Z., Wei J.-J., Wu X.-F., Liang E.-W., 2016, ArXiv e-prints

Ellis R. S. et al., 2013, ApJ, 763, L7

Falcone A. D. et al., 2007, ApJ, 671, 1921

Fenimore E. E., Ramirez-Ruiz E., 2000, ArXiv Astrophysics e-prints

Firmani C., Avila-Reese V., Ghisellini G., Tutukov A. V., 2004, ApJ, 611, 1033

Firmani C., Ghisellini G., Avila-Reese V., Ghirlanda G., 2006, MNRAS, 370, 185

Fryer C. L., Woosley S. E., Hartmann D. H., 1999, ApJ, 526,152

G. Dainotti M., Del Vecchio R., Shigehiro N., Capozziello S., 2014, ArXiv e-prints

Galama T. J. et al., 1998, Nature, 395, 670

Gehrels N. et al., 2004, ApJ, 611, 1005

Gelman A., Rubin D. B., 1992, Statistical Science, 7, 457

Genet F., Granot J., 2009, MNRAS, 399, 1328

Goldstein A., Preece R. D., Mallozzi R. S., Briggs M. S., Fishman G. J., Kouveliotou C., Paciesas W. S., Burgess J. M., 2013, ApJS, 208, 21 
Gompertz B. P., O'Brien P. T., Wynn G. A., 2014, MNRAS, 438, 240

Guetta D., Piran T., Waxman E., 2005, ApJ, 619, 412

Guiriec S. et al., 2011, ApJ, 727, L33

Hogg D. W., 1999, ArXiv Astrophysics e-prints

Hopkins A. M., Beacom J. F., 2006, ApJ, 651, 142

Howell E. J., Coward D. M., 2013, MNRAS, 428, 167

Jones E., Oliphant T., Peterson P., 2001, SciPy: Open source scientific tools for Python. [Online; accessed 201607-04]

Kistler M. D., Yüksel H., Beacom J. F., Stanek K. Z., 2008, ApJ, 673, L119

Kobayashi M. A. R., Inoue Y., Inoue A. K., 2013, ApJ, 763, 3

Kocevski D., Liang E., 2006, ApJ, 642, 371

Kouveliotou C., Meegan C. A., Fishman G. J., Bhat N. P., Briggs M. S., Koshut T. M., Paciesas W. S., Pendleton G. N., 1993, ApJ, 413, L101

Krimm H. A. et al., 2009, ApJ, 704, 1405

Kroupa P., 2001, in Astronomical Society of the Pacific Conference Series, Vol. 228, Dynamics of Star Clusters and the Milky Way, Deiters S., Fuchs B., Just A., Spurzem R., Wielen R., eds., p. 187

Langer N., Norman C. A., 2006, ApJ, 638, L63

Larson D. et al., 2011, ApJS, 192, 16

Li L.-X., 2008, MNRAS, 388, 1487

Liang E., Zhang B., Virgili F., Dai Z. G., 2007, ApJ, 662, 1111

Lloyd-Ronning N. M., Fryer C. L., Ramirez-Ruiz E., 2002, ApJ, 574, 554

MacFadyen A. I., Woosley S. E., 1999, ApJ, 524, 262

Mészáros P., 2006, Reports on Progress in Physics, 69, 2259

Natarajan P., Albanna B., Hjorth J., Ramirez-Ruiz E., Tanvir N., Wijers R., 2005, MNRAS, 364, L8

Norris J. P., Bonnell J. T., 2006, ApJ, 643, 266

Norris J. P., Bonnell J. T., Kazanas D., Scargle J. D., Hakkila J., Giblin T. W., 2005, ApJ, 627, 324

Norris J. P., Marani G. F., Bonnell J. T., 2000, ApJ, 534, 248

Oesch P. A. et al., 2013, ApJ, 773, 75

Paczyński B., 1998, ApJ, 494, L45

Pérez-González P. G. et al., 2005, ApJ, 630, 82

Pescalli A. et al., 2015, ArXiv e-prints

Petrosian V., Kitanidis E., Kocevski D., 2015, ArXiv eprints

Porciani C., Madau P., 2001, ApJ, 548, 522

Qin S.-F., Liang E.-W., Lu R.-J., Wei J.-Y., Zhang S.-N., 2010, MNRAS, 406, 558

Reichart D. E., Lamb D. Q., Fenimore E. E., Ramirez-Ruiz E., Cline T. L., Hurley K., 2001, ApJ, 552, 57

Robertson B. E., Ellis R. S., 2012, ApJ, 744, 95

Rujopakarn W. et al., 2010, ApJ, 718, 1171

Sakamoto T. et al., 2008, ApJS, 175, 179

Salvaterra R. et al., 2012, ApJ, 749, 68

Salvaterra R., Chincarini G., 2007, ApJ, 656, L49

Salvaterra R., Guidorzi C., Campana S., Chincarini G., Tagliaferri G., 2009, MNRAS, 396, 299

Schmidt M., 2001, ApJ, 552, 36

Scholz F. W., Stephens M. A., 1987, Journal of the American Statistical Association, 82, 918

Stanek K. Z. et al., 2003, ApJ, 591, L17
T. W. Anderson D. A. D., 1954, Journal of the American Statistical Association, 49, 765

van Dokkum P. G., 2008, ApJ, 674, 29

Virgili F. J., Liang E.-W., Zhang B., 2009, MNRAS, 392, 91

Virgili F. J., Zhang B., Nagamine K., Choi J.-H., 2011, MNRAS, 417, 3025

Wanderman D., Piran T., 2010, MNRAS, 406, 1944

Wei D. M., Gao W. H., 2003, MNRAS, 345, 743

Wilkins S. M., Trentham N., Hopkins A. M., 2008, MNRAS, 385, 687

Willingale R., Genet F., Granot J., O'Brien P. T., 2010, MNRAS, 403, 1296

Woosley S. E., 1993, ApJ, 405, 273

Yonetoku D., Murakami T., Nakamura T., Yamazaki R., Inoue A. K., Ioka K., 2004, ApJ, 609, 935

Yu H., Wang F. Y., Dai Z. G., Cheng K. S., 2015, ArXiv e-prints

Zheng W. et al., 2012, Nature, 489, 406 